\title{
Homology modeling of major intrinsic proteins in rice, maize and Arabidopsis: comparative analysis of transmembrane helix association and aromatic/arginine selectivity filters
} Anjali Bansal and Ramasubbu Sankararamakrishnan*

Address: Department of Biological Sciences and Bioengineering, Indian Institute of Technology, Kanpur 208 016, India

Email: Anjali Bansal - anjalib@iitk.ac.in; Ramasubbu Sankararamakrishnan* - rsankar@iitk.ac.in

* Corresponding author

Published: 19 April 2007

BMC Structural Biology 2007, 7:27 doi:10.1 186/1472-6807-7-27

This article is available from: http://www.biomedcentral.com/1472-6807/7/27

This is an Open Access article distributed under the terms of the Creative Commons Attribution License (http://creativecommons.org/licenses/by/2.0), which permits unrestricted use, distribution, and reproduction in any medium, provided the original work is properly cited.

\begin{abstract}
Background: The major intrinsic proteins (MIPs) facilitate the transport of water and neutral solutes across the lipid bilayers. Plant MIPs are believed to be important in cell division and expansion and in water transport properties in response to environmental conditions. More than 30 MIP sequences have been identified in Arabidopsis thaliana, maize and rice. Plasma membrane intrinsic proteins (PIPs), tonoplast intrinsic proteins (TIPs), Nod26-like intrinsic protein (NIPs) and small and basic intrinsic proteins (SIPs) are subfamilies of plant MIPs. Despite sequence diversity, all the experimentally determined structures belonging to the MIP superfamily have the same "hourglass" fold.
\end{abstract}

Results: We have structurally characterized 39 rice and 31 maize MIPs and compared them with that of Arabidopsis. Homology models of 105 MIPs from all three plant species were built. Structurebased sequence alignments were generated and the residues in the helix-helix interfaces were analyzed. Small residues (Gly/Ala/Ser/Thr) are found to be highly conserved as a group in the helixhelix interface of MIP structures. Individual families sometimes prefer one or another of the residues from this group. The narrow aromatic/arginine (ar/R) selectivity filter in MIPs has been shown to provide an important constriction for solute permeability. Ar/R regions were analyzed and compared between the three plant species. Seventeen TIP, NIP and SIP members from rice and maize have ar/R signatures that are not found in Arabidopsis. A subgroup of rice and maize NIPs has small residues in three of the four positions in the ar/R tetrad, resulting in a wider constriction. These MIP members could transport larger solute molecules.

Conclusion: Small residues are group-conserved in the helix-helix interface of MIP structures and they seem to be important for close helix-helix interactions. Such conservation might help to preserve the hour-glass fold in MIP structures. Analysis and comparison of ar/R selectivity filters suggest that rice and maize MIPs could transport more diverse solutes than Arabidopsis MIPs. Thus the MIP members show conservation in helix-helix interfaces and diversity in aromatic/arginine selectivity filters. The former is related to structural stability and the later can be linked to functional diversity. 


\section{Background}

Aquaporins and aquaglyceroporins belong to the ancient superfamily of Major Intrinsic Proteins (MIPs) and facilitate passive transport of water and small solutes across membranes of various organisms [1,2]. Aquaporins are likely to have important role in growth, development and stress response in plants [3-5]. MIPs in plants are abundant and constitute a large and highly divergent protein family. For instance, Arabidopsis thaliana and Zea mays have more than 30 aquaporin-encoding genes each $[6,7]$. Phylogenetic analysis of plant MIP sequences revealed four major subfamilies: the plasma membrane intrinsic proteins (PIPs), tonoplast intrinsic proteins (TIPs), nodulin 26-like intrinsic proteins (NIPs) and small and basic intrinsic proteins (SIPs). Functionally, two main categories have been well established for MIPs from mammals: water-specific aquaporins and solute-transporting aquaglyceroporins [2]. Most of the plant MIPs that have been investigated are highly specific for water. Several TIPs have been reported to facilitate the transport of urea and ammonia and NIPs have been shown to transport glycerol [8-12]. Recent studies indicate that PIPs contribute to $\mathrm{CO} 2$ diffusion across leaf tissues [13]. The substrate specificity of SIP proteins has not been studied in detail.

The primary sequence of aquaporins exhibits an internal homology and there is $\sim 20 \%$ conservation between the $\mathrm{N}$ and the C-terminal halves [14]. The high-resolution structures of several members of MIP family have been determined and are available in the Protein Data Bank (PDB) [15]. Bovine AQP1 [16], GlpF [17] and AqpZ [18] from E. coli, sheep and bovine AQP0 $[19,20]$, SoPIP2; 1 from spinach [21] and the archaeal aquaporin AqpM from Methanothermobacter marburgensis [22], all reveal a homotetrameric organization and each aquaporin monomer forms an independent functional pore. This canonical fold is characterized by a six tilted, membranespanning (TM1 to TM6) right-handed helical bundle connected by five loop regions (loops $\mathrm{A}$ to $\mathrm{E}$ ) with $\mathrm{N}$ and $\mathrm{C}$ terminal ends located on the cytoplasmic side of the membrane (Figure 1). Loops B and E contain the highly conserved NPA (Asn-Pro-Ala) motifs and form two halfhelices that dip into the membrane from opposite sides. The N-terminal ends of these half-helices are connected by interactions between the two NPA boxes and it is also one of the two major constrictions in the channel $[1,14]$. The second major constriction is located $\sim 8 \AA$ above the NPA region towards the periplasmic side (Figure 1) and this primary selectivity filter known as the aromatic/ arginine $(\mathrm{ar} / \mathrm{R})$ region is formed by two residues from TM2 and TM5 and two residues from loop E $[1,14]$. This constriction has been shown as a major check point for solute permeability [23].
The ar/R selectivity filter of spinach plasma membrane intrinsic protein SoPIP2; 1 (Figure 2) and three other highresolution water-specific aquaporin structures, AQP1 [16], AqpZ [18], and AQP0 [19,20], contain a Phe from TM2 (H2 position), His from TM5 (Position H5) and Arg from the loop E (LE2). A fourth residue from loop E (LE1 position) also forms part of the selectivity region by providing its backbone carbonyl oxygen to the ar/R filter and this is usually observed to be a small residue (Cys, Thr or Ala). Crystal structures indicate that His and Arg of ar/R region could provide donor hydrogen bonds for water molecules. The ar/R selectivity filters of Arabidopsis PIP members resemble that of water-selective mammalian and microbial aquaporins [24]. In the glycerol-specific GlpF [17], ar/R selectivity filter residues Phe (H2), His (H5) and the smaller residue at LE1 position typically found in water-sepcific channels are substituted by Trp, Gly and Phe respectively (Figure 2). This gives rise to the selectivity filter with larger pore diameter among the

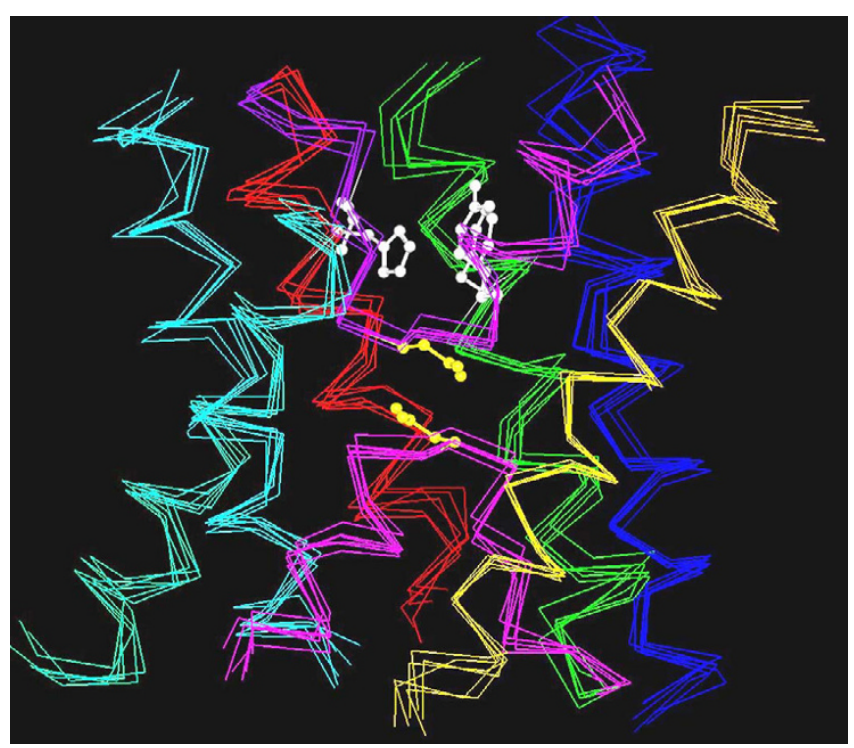

Figure I

Superposition of aquaporin crystal structures. The transmembrane regions of six aquaporin crystal structures (bovine AQPI, E. coli AqpZ, sheep AQP0, spinach plasma membrane aquaporin SoPIP2; I, archaeal aquaporin AqPM from Methanothermobacter marburgensis and E. coli GlpF) are superposed. The corresponding PDB IDs are IJ4N, IRC2 (B chain), 2B6O, IZ98 (A chain), 2F2B and IFX8 respectively. For clarity, $\mathrm{C} \alpha$ traces of only the six transmembrane helices and the loops $B$ and $E$ are shown: TMI - blue, TM2 - green, loop B - pink, TM3 - orange, TM4 - red, loop E - purple, TM5 - cyan and TM6 - green. The residues forming the Ar/R selectivity filter from SoPIP2; I are shown in white and the asparagines from the conserved NPA motif of loops B and E are shown in yellow. The aquaporin structures from bacteria, archaea, plant and mammals show a conserved "hour-glass" fold and the helices form a right-handed bundle structure. 
known aquaporin structures with two hydrophobic walls opposite the conserved arginine. Such architecture is thought to facilitate the transport of glycerol efficiently [25-28].

Draft genome sequences of rice and more recently, highly accurate finished rice genome sequence have been determined [29-31]. In this paper, we have obtained the complete set of rice aquaporins (OsMIPs) from the rice genomic sequence. We have built three-dimensional structures of rice (OsMIPs) and maize (ZmMIPs) MIPs using homology modeling with AQP1, GlpF and AqpZ crystal structures as templates. Their models were compared with that of Arabidopsis (AtMIPs) MIPs. It has been realized that conserved glycines within the transmembrane helices facilitate the closest approaches of helices in the center of the aquaporin helix bundle $[18,32,33]$. We
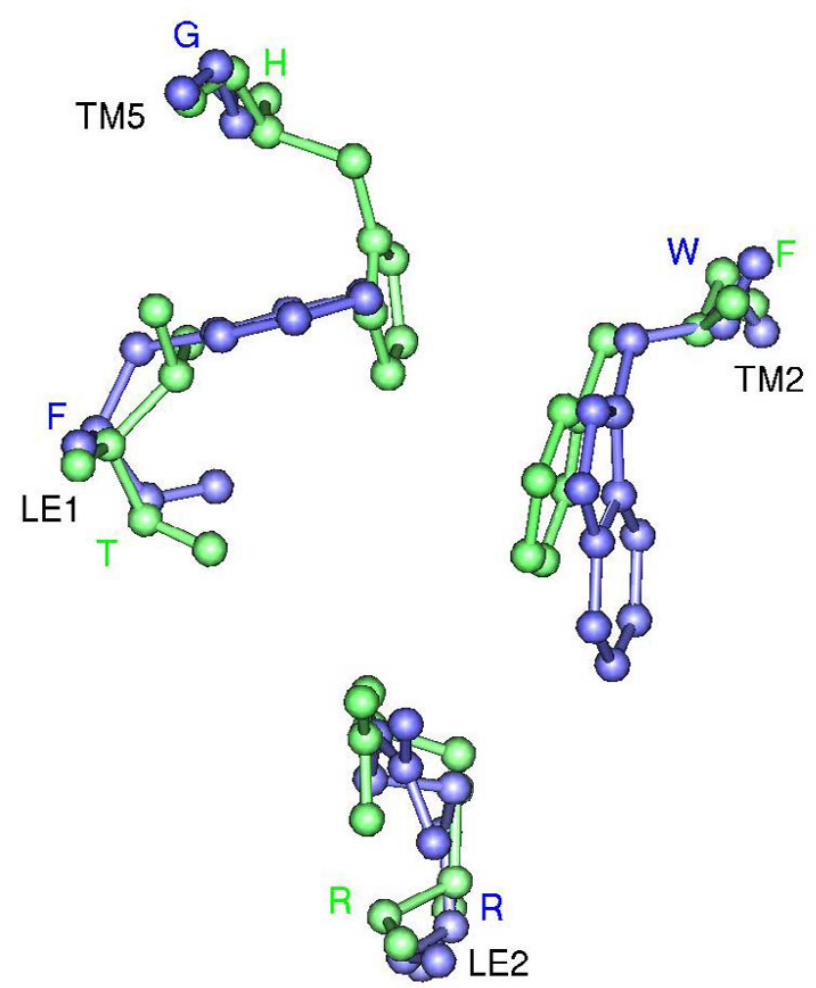

\section{Figure 2}

Ar/R selectivity filters of SoPIP2; I and GIpF. Ar/R selectivity filter of water-specific SoPIP2; I (green) and glycerol specific GlpF (blue). Transmembrane regions of both structures were first superposed and only the residues forming the ar/R tetrad from the superposed structures are shown in ball-and-stick model. Residue names in one letter code are given for SoPIP2; I in green and for GlpF in blue. The transmembrane segments and the loop regions to which these residues belong are indicated. The projection shown for each filter is viewed perpendicular to the membrane plane from the extracellular side. have generated structure-based sequence alignments in the transmembrane region of all the plant MIPs from the homology models. Conservation of residues in the helixhelix interfaces was analyzed and our results show that residues occurring in the helix-helix interfaces are small and are strongly group-conserved in plant MIPs. Wallace and Roberts [24] have recently performed sequence alignments and homology modeling studies of AtMIPs. Based on the residues that form the ar/R selectivity filter, it was found that TIP, NIP and SIP families diverge from the classical aquaporin structures. Their results suggested that these proteins are likely to have functions distinct from classical aquaporins and aquaglyceroporins. Hence we compared the ar/R selectivity filters of rice and maize with that of Arabidopsis. Analysis of pore selectivity regions reveals that NIPs and TIPs from rice and maize are much more diverse compared to AtMIPs. Based on the structural analysis, we have identified potential MIP candidates that could possibly transport diverse solute molecules such as arsenite.

\section{Results \\ Rice MIP sequences}

Identification of rice MIP sequences has become possible due to the availability of complete rice genome sequence. TBLASTN [34-37] searches made in GenBank [38,39], Rice Genome Project (RGP) [40] and The Institute of Genomic Research (TIGR) $[41,42]$ found thirty nine different rice MIP genes. Recently, Sakurai et al. [43] have reported the identification of 33 rice MIP genes and investigated their expression and function. In their study, classification based on phylogenetic analysis indicated the presence of 11 PIPs, 10 TIPs, 10 NIPs and 2 SIPs. Sequence comparisons showed that all the reported 33 MIP genes are identified in our analysis also. We included the six additional MIPs (Table 1) and performed phylogenetic analyses of all 39 proteins (Figure 3 ). The groups recognized in earlier analyses are more or less intact in the present study. Hence the nomenclature for the 33 sequences is retained as reported by Sakurai et al. [43]. Among the six additional MIPs, there are 2 PIPs, one TIP and 3 NIPs and their names are given based on the subfamily they belong to. The additional PIPs identified in this study are the two mRNA sequences of indica-cultivar group available from the GenBank (only these two are from the indica subspecies and all other rice MIPs in this study belong to the japonica subspecies). OsTIP2;3 has a very long C-terminal extension and hence we have considered only the aquaporin region in this sequence for further analysis. Two of the three NIP sequences have been identified from TIGR release 2 and their amino acid sequences have undergone changes from Release 2 to Release 4. In OsNIP3;4, N-terminus is longer by 43 residues in Release 4. A large deletion is observed in OsNIP1;5 between the two NPA regions. However, pre- 
diction of amino acid sequence from genomic sequence using GeneMark [44] supports the sequences from Release 2. Hence in the present study, we have used amino acid sequences from Release 2 for OsNIP1;5 and OsNIP3;4. All the six additional sequences were correctly recognized as $\mathrm{MIP} /$ aquaporin sequences in Interpro $[45,46]$ and the rice PIP and TIP sequences identified in this work (OsPIP1;4, OsPIP1;5 and OsTIP2;3) were also found in the database of MIP family of proteins $[47,48]$. Homology models of 39 rice MIPs, 31 maize MIPs were built as per the modeling protocol described in the Methods section. The same procedure was also used to construct the models of Arabidopsis MIPs. In total, 105 models were used to analyze the residue conservation in helixhelix interface and the nature of the ar/R selectivity filters in plant MIPs.

\section{Residues in the helix-helix interfaces of MIP family members}

Table 2 lists the residue pairs occurring at the interfaces of pairs of TM helices or of a TM helix and the half-helix from loops B/E of all aquaporin crystal structures. These residue pairs are within $3.5 \AA$ of the interacting helix in at least one of the template structures used in the modeling study. Such interactions are between either side chain side chain or side chain - back bone atoms and most are observed between three helix pairs: TM1 and TM3, TM4 and TM6, and, TM2 and TM5 (Figure 4). The sequence motifs GAxxA/GGxxA/GAxxG from TM3, GxxxGAxxA/ GxxxGAxxG from TM6 and SxxxG/AxxxG/GxxxG from TM2 have close $C \alpha-C \alpha$ contacts $(\leq \sim 6 \AA)$ with helices TM1, TM4 and TM5 respectively. The interface of TM1 and TM2 is also characterized by interactions between a small residue from TM1 (Thr 55 from 1Z98 and equivalent residues from other structures; see Table 2) and the residue that participates in the ar/R selectivity filter from TM2. Additionally, close contacts between the half-helices from loops B and E are observed with TM6 and TM3 respectively. The average distances between C $\alpha$ atoms of equivalent residue pairs calculated from the six high-resolution crystal structures are also given in Table 2. Most of the distances are less than $6 \AA$ indicating a very close approach of the helix pairs, TM1-TM2, TM1-TM3, TM4-TM6 and TM2-TM5. At least one residue occurring at the helix-helix interfaces can be classified as small and weakly polar residue (Gly, Ala, Ser and Thr). In three cases (TM2-TM5, LBTM6, LE-TM3), both residues in the interfaces are small. In total, seventeen small and weakly polar residues seem to play an important role in close helix packing in known aquaporin structures (Table 2). We have studied the conservation of these residues in the structure-based sequence alignments of all plant MIPs and also in each of the subfamilies separately.

The occurrence and the role of small and weakly polar residues in helix-helix interfaces of membrane proteins have been extensively investigated in earlier studies [33,49]. Analysis of high-resolution structures of $\alpha$-helix bundle proteins revealed that high abundance of small residues (Gly, Ser, Thr and Ala) mediate helix-helix interactions in membrane proteins and result in closely packed helices. Hence, for the purpose of this analysis, the residues Gly, Ser, Thr, Ala and Cys have been grouped together and their group conservation is determined in the interfacial positions. We have also reported the individual residues from this group and their conservation if it exceeds $25 \%$ (Table 3 ). All the 17 positions are more than $90 \%$ groupconserved in the 105 MIP sequences. Group-conservation for small residues for the helix interfacial positions is the highest (97-100\%) for the PIP sub-family followed by the TIP members (94-100\%). Although, majority of the small residues are highly conserved in NIP family members, the conservation of Thr 55 and Ala 253 (residues and their corresponding residue numbers reported in this section are from $1 \mathrm{Z98}$ unless otherwise mentioned) falls below $90 \%$ in these two positions. Group-conservation of small residues in helix-helix interface is generally high in SIP family, but some positions are poorly conserved (Ala 78, Gly 82 and Ser 181). Many important positions that are likely to affect the interior properties of the channels have been shown to be different in SIP members in comparison with other plant MIP proteins [50]. It should also be noted that the sample size for SIP family is very small (8 members).

While the group-conservation is very strong in all the 17 interfacial positions, there are instances in which subfamilies show strong preference for one or another amino

Table I: Six additional rice MIP sequences. Details of new rice MIP genes identified from the TBLASTN search.

\begin{tabular}{lccc}
\hline Name & Accession no. $^{\text {a }}$ & Length & Comments \\
\hline OsPIPI;4 & AAB82140 & 289 & Belongs to indica cultivar-subspecies \\
OsPIPI;5 & $\underline{\text { AABI8817 }}$ & 291 & Belongs to indica cultivar-subspecies \\
OsTIP2;3 & CAD41593 & 768 & Has $~ 500$ residue long C-terminal extension \\
OsNIPI;5 & $9629 . t 00960$ & 303 & TIGR Release 4 has 70 residue deletion between the two NPA regions \\
OsNIP3;4 & $9640 . t 00931$ & 267 & N-terminus is longer by 3I residues in TIGR release 4 \\
OsNIP3;5 & BAC98553 & 283 & \\
\hline
\end{tabular}

a Accession codes of NIPI;5 and NIP3;4 are from TIGR; all others are GenBank accession codes 


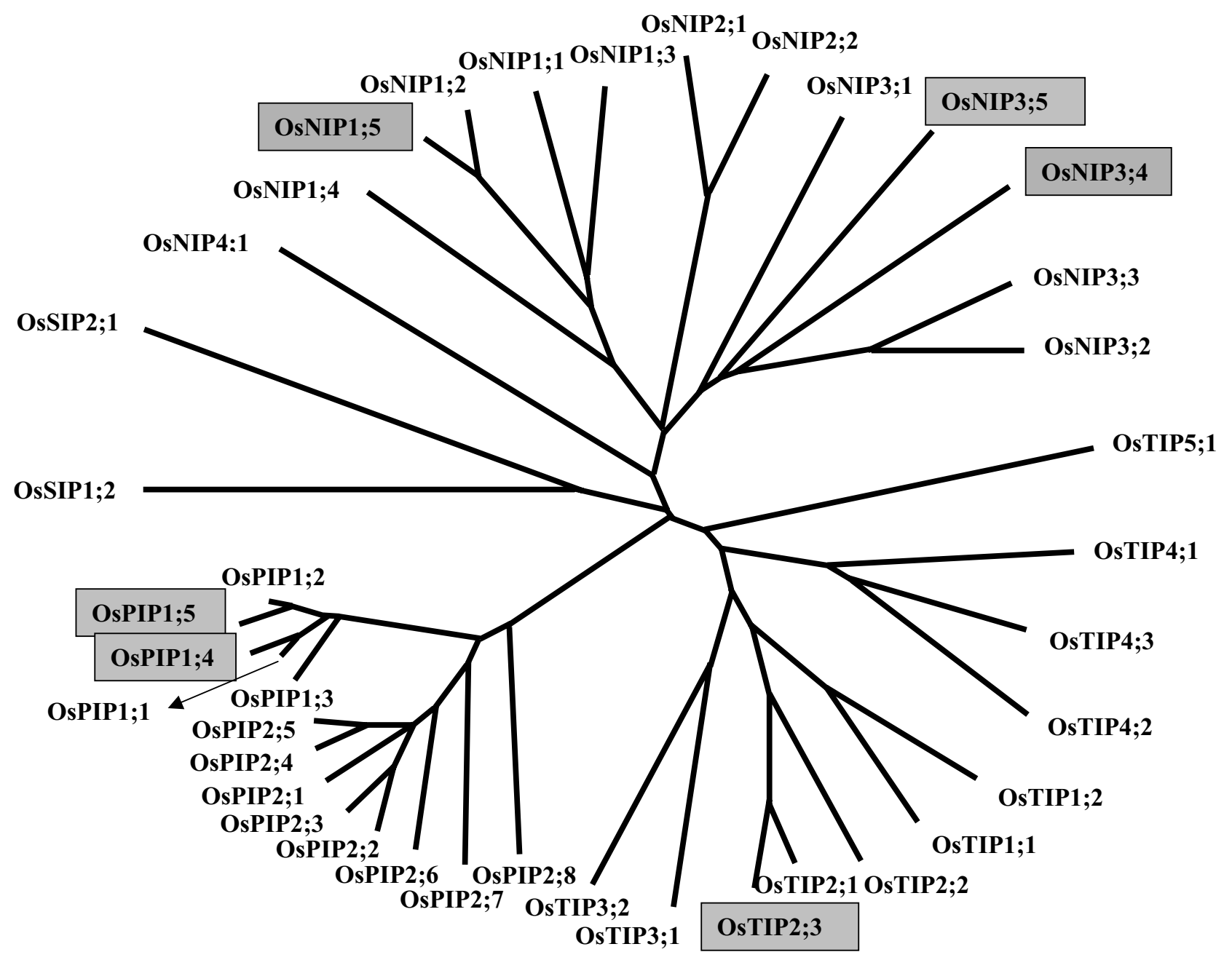

0.1

Figure 3

Additional rice MIPs in the phyologenetic tree of all rice MIPs. Phylogenetic analysis of all 39 rice MIP sequences is shown. This tree was created using the Neighbor-Joining method and the multiple sequence alignment for this purpose was generated by the T-Coffee program [8I]. As observed in Arabidopsis and maize, rice MIPs also can be classified into four subfamilies. OsPIPs, OsTIPs, OsNIPs and OsSIPs respectively indicate plasma membrane intrinsic proteins, tonoplast intrinsic proteins, Nod26-like intrinsic proteins and small basic intrinsic proteins from rice. Thirty three out of thirty nine sequences have been identified by Sakurai et al. [43]. The additional six sequences identified in this study are shown within gray boxes.

acid of this group (see Additional files 1, 2, 3, 4, 5, 6, 7, 8). For instance, glycine is strongly conserved at positions 82 and 133 in PIPs, but alanine is the preferred amino acid for TIPs in the same position (Table 3). Similarly, alanine is strongly conserved at position 256 in PIPs and TIPs, but glycine has $100 \%$ conservation at this position in NIP members.

\section{Comparison of the Ar/R selectivity filters in rice/maize and Arabidopsis}

Wallace and Roberts [24] have recently performed sequence alignments and homology modeling studies of
AtMIPs. Based on the residues that form the ar/R selectivity filter, Arabidopsis aquaporins have been classified into eight structural subclasses. The amino acid signatures of subclasses belonging to TIP, NIP and SIP families diverge from the classical aquaporin structures and it has been suggested that these proteins are likely to have functions distinct from classical aquaporins and aquaglyceroporins. $\mathrm{Ar} / \mathrm{R}$ selectivity filter was analyzed in all the homology models generated in this study and compared among the three plant species. All the PIP members from the three plant species have ar/R selectivity filter that shows similarity with water-transporting AQP1. Aromatic/arginine sig- 
Table 2: Residues in the helix-helix interfaces of aquaporin crystal structures. Residue pairs that occur at the helix-helix interface in the six high-resolution aquaporin crystal structures.

\begin{tabular}{|c|c|c|}
\hline Helix Pairs & Residues from the crystal structures ${ }^{a}$ & $<$ dist. $>$ b $(\AA)$ \\
\hline TMI...TM2 & 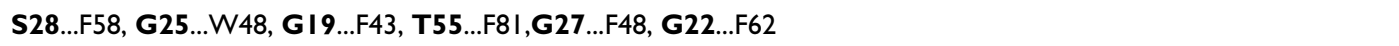 & 4.86 \\
\hline \multirow[t]{4}{*}{ TMI...TM3 } & 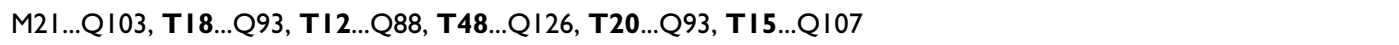 & 5.74 \\
\hline & 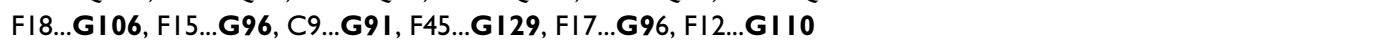 & 4.55 \\
\hline & 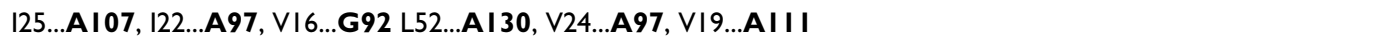 & 5.70 \\
\hline & 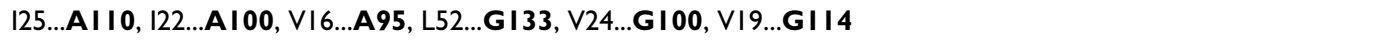 & 5.52 \\
\hline \multirow[t]{6}{*}{ TM4...TM6 } & 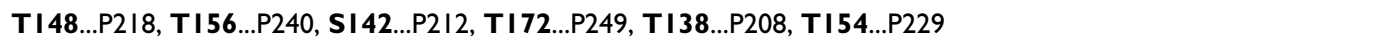 & 5.29 \\
\hline & 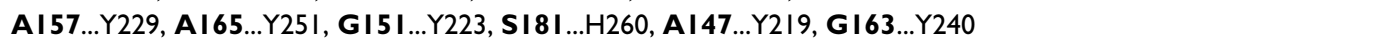 & 7.73 \\
\hline & 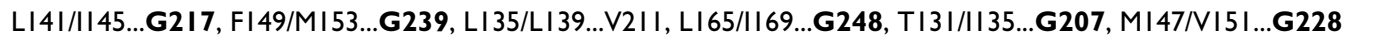 & 5.31 \\
\hline & 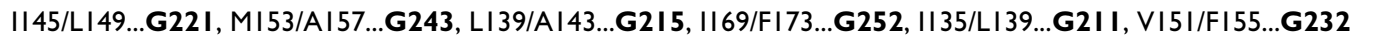 & 4.35 \\
\hline & 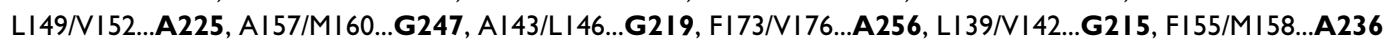 & 5.17 \\
\hline & 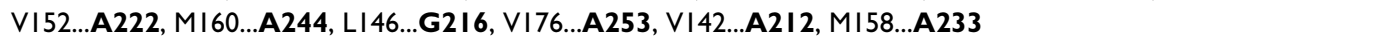 & 5.51 \\
\hline \multirow[t]{2}{*}{ TM2...TM5 } & 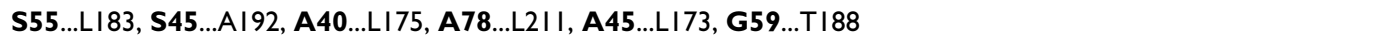 & 5.91 \\
\hline & 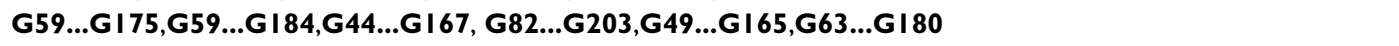 & 4.31 \\
\hline LB...TM4 & 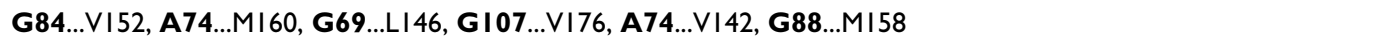 & 6.96 \\
\hline LB...TM6 & 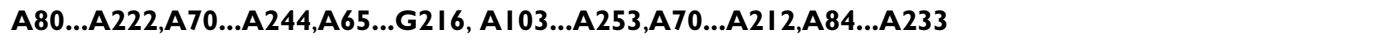 & 5.75 \\
\hline LE...TM3 & 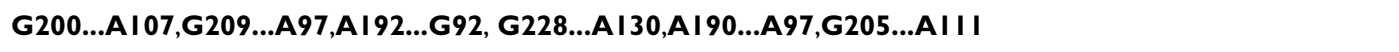 & 4.89 \\
\hline LE...TM6 & 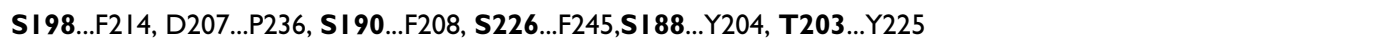 & 6.52 \\
\hline
\end{tabular}

a Residue pairs are given respectively for the crystal structures $\underline{\mathrm{J}} 4 \mathrm{~N}, \underline{I F X} 8, \underline{I R C 2}, \underline{I Z 98}, \underline{2 B} 60$ and $\underline{2 F} 2 \mathrm{~B}$. For each residue given for $1 \mathrm{~J} 4 \mathrm{~N}$, the equivalent residues from the other crystal structures were obtained from structure-based sequence alignment (see Additional files I to 8 ). For example, the equivalent residue pairs for $(S 28, F 58)$ in $\underline{J} 4 \mathrm{~N}$ are $(\mathrm{G} 25, \mathrm{~W} 48)$ in $\underline{\mathrm{IFX}}$, (GI9, F43) in $\underline{I R C 2},(\mathrm{T55}, \mathrm{F} 8 \mathrm{I})$ in $\underline{\mathrm{IZ} 98},(\mathrm{G} 27, \mathrm{~F} 48)$ in $\underline{2 B 60}$ and $(\mathrm{G} 22, \mathrm{~F} 62)$ in $2 \mathrm{~F} 2 \mathrm{~B}$. Small residues (Gly/Ala/Ser/Thr) are shown in bold. ${ }^{\mathrm{b}}$ Average $\mathrm{C} \alpha$...C $\alpha$ distances calculated for residue pairs from all the six crystal structures are given.

natures of 30 out of 39 OsMIPs and 23 out of 31 ZmMIPs are identical or similar to their Arabidopsis counterparts (Table 4). The characteristics of these selectivity filters have been described in detail in the earlier studies [24] and hence will not be discussed here. MIP members that have unique ar/R selectivity filters found only in rice and maize are discussed in detail below.

Analysis of homology models shows that some rice and maize TIP family members have ar/R selectivity filters that are not found in Arabidopsis and they are listed in Table 5 along with the residues in the four positions that form the $\mathrm{ar} / \mathrm{R}$ signatures. In five TIPs from maize (ZmTIP4;1, ZmTIP4;2 and ZmTIP4;3) and rice (OsTIP4;1 and OsTIP4;2), the H5 position contains a small hydroxyl residue. In these members, the ar/R selectivity filter is devoid of any hydrophobic residue at both $\mathrm{H} 2$ and $\mathrm{H} 5$ positions, thus making it highly polar. The LE1 and LE2 positions in this group are occupied by Ala and the highly conserved Arg residue respectively. No Arabidopsis TIP gene has an ar/ $\mathrm{R}$ filter with this feature (Table 5). The selectivity filter of representative member of this group, OsTIP4;2 is shown in Figure 5. The pore diameter at the ar/R selectivity filter region for this model is very similar to that of GlpF (Figure 6), but this constriction is more hydrophilic. In OsTIP4;1, Thr is found at both $\mathrm{H} 2$ and $\mathrm{H} 5$ positions and as a result this will have a larger pore diameter at the constriction region. In OsTIP5;1 and ZmTIP5;1, the H5 position has Val and hence the selectivity filter is less hydrophilic than the other members from this group.

Small residues (Gly/Ala/Ser/Cys) at $\mathrm{H} 2$ and $\mathrm{H} 5$ positions are found in seven rice (OsNIP2;1, OsNIP2;2, OsNIP3;2, OsNIP3;5 and OsNIP4;1) and maize (ZmNIP2;1 and ZmNIP2;2) NIP members. Except OsNIP3;5, the position LE1 also possesses a small residue (Gly/Ala) in these members. OsNIP3; 5 has a proline in this position. Due to the small size of the residues that form the selectivity filter, these NIP members are likely to have the constriction size that is the largest among all the generated homology models. Residues forming the selectivity filter are shown for a representative member of this group (Figure 5). Due to the small size of the residues, the pore diameter in this region is about $1 \AA$ larger compared to GlpF and about 2.2 $\AA$ larger than the water-specific plant PIP structure (Figure 6). 

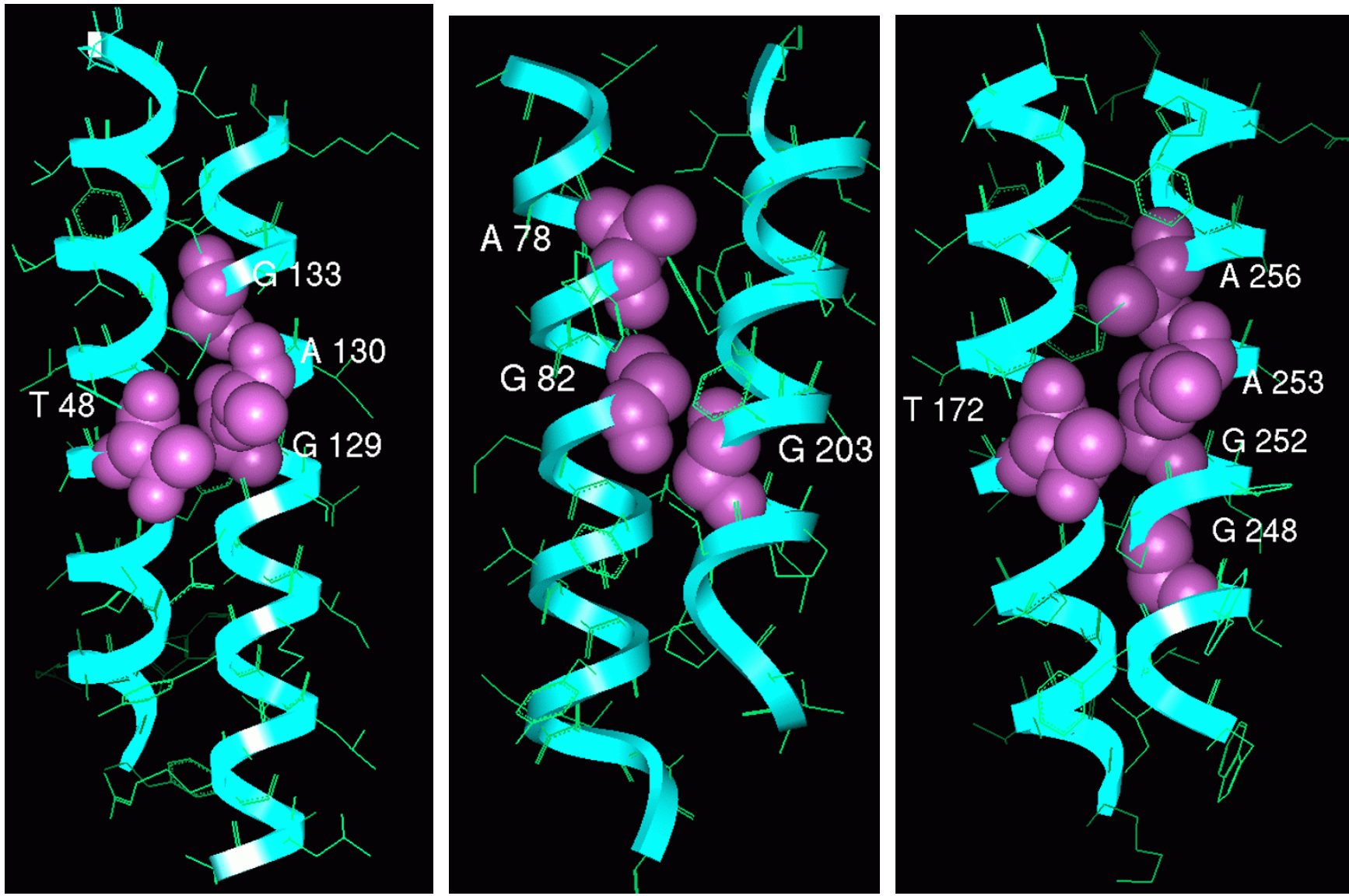

Figure 4

Small and polar residues at the helix-helix interfaces of SoPIP2; I. Small and polar residues occurring in helix-helix interfaces of spinach plant aquaporin SoPIP2;I are displayed. The helix pairs TMI-TM3 (left), TM2-TM5 (middle) and TM4TM6 (right) are shown. The backbone is drawn in ribbon representation and the interfacial residues are depicted as space-filling models. Residue numbers of interfacial residues correspond to the PDB structure $\underline{\text { IZ98. }}$.

SIP family members are more distantly related to other MIP members and also with the three template sequences. The selectivity filters of SIP members differ significantly from other plant MIP family and any of the known AQP members. For the two Arabidopsis members, AtSIP1;1 and AtSIP1;2, the putative ar/R signature obtained in this study (Table 4) differs from the earlier models created by Wallace and Roberts [24]. The H2 position contains a hydrophobic residue (Ile/Val) instead of Thr. Residues in other positions are observed to be the same and hence hydrophobic character of this filter in our model will be greater than that reported in the previous study. In SIP members OsSIP1;1, ZmSIP1;1 and ZmSIP1;2, both H2 and $\mathrm{H} 5$ positions are hydrophobic and Pro and Asn are observed in loop E positions.

\section{Discussion}

\section{Reliability of the plant MIP homology models}

In this study, we have modeled 105 plant MIP sequences from three different plant species. The initial and an important step in comparative modeling is the selection of the template structure(s). The higher the sequence identity between the sequence(s) of the template structure(s) and the target sequences, the most reliable will be the generated models [51]. In the present study, the sequence identity between the template structures and the target sequences is not very high ( 26 to $46 \%$ for PIPs, TIPs and NIPs; 22 to $29 \%$ for SIPs; Table 6). The experimentally determined aquaporin structures from archaea, bacteria, plant and mammals all show a remarkably conserved hour-glass model with right-handed helical bundle structure (Figure 1). For example, the RMSD is less than $1 \AA$ on C $\alpha$ atoms within the transmembrane regions between the animal and plant aquaporins [21] and the sequence identity between these sequences is $\sim 40 \%$. Based on molecular dynamics simulations, Law and Sansom [52] suggested that homology models based on bacterial homologs may be used to derive meaningful information on the structure, dynamics and function of the corresponding mammalian protein. Before modeling 
Table 3: Conservation of small and weakly polar residues at the helix-helix interfaces of plant MIP proteins

\begin{tabular}{|c|c|c|c|c|c|}
\hline Residue $^{\mathrm{a}}$ & All families $b, c$ & $\mathrm{PIPs}^{\mathrm{c}}$ & TIPsc & $\mathrm{NIPs}^{\mathrm{c}}$ & SIPsc \\
\hline T48 & $\mathrm{T}(93), \mathrm{A}, \mathrm{S}(\mathbf{9 7 \% )}$ & $\mathrm{T}(100)(100 \%)$ & $\mathrm{T}(94)(94 \%)$ & $\mathrm{T}(92), \mathrm{S}(96 \%)$ & $\mathrm{T}(63), \mathrm{A}(25), \mathrm{S}(100 \%)$ \\
\hline T55 & G(39), T(35), A (93\%) & $\mathrm{T}(85), \mathrm{S}(100 \%)$ & $\mathrm{G}(78), \mathrm{A}(100 \%)$ & $\mathrm{G}(50), \mathrm{T}, \mathrm{A}(8 \mathrm{I} \%)$ & $\mathrm{G}(38), \mathrm{S}(25), \mathrm{A}(75 \%)$ \\
\hline A78 & $\mathrm{A}(77), \mathrm{S}, \mathrm{C}, \mathrm{T}(93 \%)$ & $A(95), S(98 \%)$ & $\mathrm{A}(88), \mathrm{S}(100 \%)$ & $A(62), C, S(96 \%)$ & $\mathrm{T}(38)(38 \%)$ \\
\hline G82 & $\mathrm{G}(63), \mathrm{A}(28), \mathrm{S}, \mathrm{T}(94 \%)$ & $\mathrm{G}(100)(100 \%)$ & $\mathrm{A}(9 \mathrm{I}), \mathrm{G}, \mathrm{T}(100 \%)$ & $\mathrm{G}(96)(96 \%)$ & $S(38)(38 \%)$ \\
\hline Al03 & $\mathrm{A}(88), \mathrm{S}, \mathrm{T}, \mathrm{C}(96 \%)$ & $\mathrm{A}(100)(100 \%)$ & $A(97)(97 \%)$ & $\mathrm{A}(85), \mathrm{S}(15)(100 \%)$ & $\mathrm{T}(50), \mathrm{C}(62 \%)$ \\
\hline G107 & $\mathrm{G}(6 \mathrm{I}), \mathrm{A}(32), \mathrm{S}, \mathrm{T}(100 \%)$ & $\mathrm{G}(97), \mathrm{A}(100 \%)$ & G(78), A, S (100\%) & $A(77), T, S, G(100 \%)$ & $A(100)(100 \%)$ \\
\hline GI29 & $\mathrm{G}(90), \mathrm{A}(100 \%)$ & $\mathrm{G}(100)(100 \%)$ & $\mathrm{G}(8 \mathrm{I}), \mathrm{A}(100 \%)$ & $G(81), A(100 \%)$ & $\mathrm{G}(100)(100 \%)$ \\
\hline $\mathrm{A} / 30$ & $A(63), S(36), G(100 \%)$ & $A(97), G(100 \%)$ & $S(59), A(4 I)(100 \%)$ & $\mathrm{S}(65), \mathrm{A}(35)(100 \%)$ & $A(75), S(25)(100 \%)$ \\
\hline GI33 & $A(55), G(44)(99 \%)$ & $\mathrm{G}(97)(97 \%)$ & $A(97), G(100 \%)$ & $A(100)(100 \%)$ & $\mathrm{G}(88), \mathrm{A}(100 \%)$ \\
\hline TI72 & $\mathrm{T}(9 \mathrm{I}), \mathrm{S}, \mathrm{A}(100 \%)$ & $\mathrm{T}(100)(100 \%)$ & $\mathrm{T}(97), \mathrm{A}(100 \%)$ & $\mathrm{T}(73), \mathrm{S}(27)(100 \%)$ & $\mathrm{T}(75), \mathrm{S}(25)(100 \%)$ \\
\hline SI8I & $\mathrm{A}(40), \mathrm{S}(40), \mathrm{G}, \mathrm{T}(93 \%)$ & $\mathrm{S}(100)(100 \%)$ & $\mathrm{A}(84), \mathrm{S}, \mathrm{T}(94 \%)$ & $\mathrm{A}(58), \mathrm{G}(35), \mathrm{S}, \mathrm{T}(100 \%)$ & $\mathrm{T}(25), \mathrm{G}(\mathbf{3 8 \%})$ \\
\hline G203 & G(9I), A, S (99\%) & $\mathrm{G}(100)(100 \%)$ & $\mathrm{G}(100)(100 \%)$ & $\mathrm{G}(92), \mathrm{A}(96 \%)$ & $S(75), A(25)(100 \%)$ \\
\hline S226 & $\mathrm{S}(7 \mathrm{I}), \mathrm{T}, \mathrm{A}(\mathbf{9 7 \% )}$ & $\mathrm{S}(97), \mathrm{T}(100 \%)$ & $S(75), A(94 \%)$ & $\mathrm{T}(54), \mathrm{S}(46)(100 \%)$ & $\mathrm{A}(88)(88 \%)$ \\
\hline G248 & $\mathrm{G}(78), \mathrm{A}, \mathrm{S}, \mathrm{C}(100 \%)$ & $G(100)(100 \%)$ & $\mathrm{G}(100)(100 \%)$ & $A(50), G(38), S(100 \%)$ & $C(38), A(25), S(25), G(100 \%)$ \\
\hline G252 & $\mathrm{G}(97), \mathrm{A}(100 \%)$ & $\mathrm{G}(100)(100 \%)$ & $\mathrm{G}(100)(100 \%)$ & $\mathrm{G}(100)(100 \%)$ & $G(62), A(38)(100 \%)$ \\
\hline A253 & $\mathrm{A}(66), \mathrm{G}, \mathrm{T}, \mathrm{S}(97 \%)$ & $A(97), G(100 \%)$ & $\mathrm{G}(72), \mathrm{A}(25), \mathrm{S}(100 \%)$ & $\mathrm{A}(69), \mathrm{T}(88 \%)$ & $\mathrm{A}(62), \mathrm{T}(38)(100 \%)$ \\
\hline A256 & $A(71), G(27)(98 \%)$ & $\mathrm{A}(97)(97 \%)$ & $A(100)(100 \%)$ & $\mathrm{G}(100)(100 \%)$ & $A(62), G(25), S(100 \%)$ \\
\hline
\end{tabular}

a Residue numbers correspond to that of the plant aquaporin SoPIP2;I (PDB ID: $\underline{\text { IZ98) }}$ ).

b Conservation is reported for all MIP subfamilies from the structure-based sequence alignments of 105 MIP sequences from rice, maize and Arabidopsis.

c Small and weakly polar interfacial residues in plant MIP sequences and their conservation (if it exceeds $25 \%$ ) are given. If the conservation is less than $25 \%$, only the residues are reported. Group conservation of small and weakly polar residues (Gly, Ala, Ser, Thr and Cys) is shown in bold and italics.

Table 4: Aromatic/Arginine signatures that are identical or similar in Arabidopsis, rice and maize

\begin{tabular}{|c|c|c|c|c|}
\hline MIP Members & $\mathrm{H} 2$ & $\mathrm{H} 5$ & LEI & LE2 \\
\hline \multicolumn{5}{|l|}{ PIP family } \\
\hline All OsPIPs All ZmPIPs All AtPIPs & $\mathrm{F}$ & $\mathrm{H}$ & $\mathrm{T}$ & $\mathrm{R}$ \\
\hline \multicolumn{5}{|l|}{ TIP family } \\
\hline OsTIPI;I, OsTIPI;2 ZmTIPI;I, ZmTIPI;2 AtTIPI;I, AtTIPI;2, AtTIPI;3 & $\mathrm{H}$ & I & A & $\vee$ \\
\hline $\begin{array}{l}\text { OsTIP2;I, OsTIP2;2, OsTIP2;3, OsTIP3;I, OsTIP3;2, OsTIP4;3 ZmTIP2;I, ZmTIP2;2, ZmTIP2;3, ZmTIP3;I, } \\
\text { ZmTIP4;4 AtTIP2;I, AtTIP2;2, AtTIP2;3, AtTIP3;I, AtTIP3;2, AtTIP4;I }\end{array}$ & $\mathrm{H}$ & $\mathrm{I} / \mathrm{M} / \mathrm{V}$ & $\mathrm{G} / \mathrm{A} / \mathrm{S}$ & $\mathrm{R}$ \\
\hline AtTIP5; $\left.\right|^{a}$ & $\mathrm{~N}$ & $\mathrm{~V}$ & G & C \\
\hline \multicolumn{5}{|l|}{ NIP family } \\
\hline $\begin{array}{l}\text { OsNIPI;I, OsNIPI;2, OsNIPI;3, OsNIPI;4, OsNIPI;5 ZmNIPI;I AtNIPI;I, AtNIPI;2, AtNIP2;I, AtNIP4;I, } \\
\text { AtNIP4;2 }\end{array}$ & W & $\vee$ & A & $\mathrm{R}$ \\
\hline OsNIP3;I, OsNIP3;3 ZmNIP3;I AtNIP5;I, AtNIP6;I, AtNIP7;I & A & $\mathrm{I} / \mathrm{V}$ & $\mathrm{A} / \mathrm{G}$ & $\mathrm{R}$ \\
\hline \multicolumn{5}{|l|}{ SIP family } \\
\hline \multirow[t]{2}{*}{ AtSIPI;I, AtSIPI; $2^{\mathrm{a}}$} & $\mathrm{I} / \mathrm{V}$ & $\mathrm{F} / \mathrm{V}$ & $P$ & I \\
\hline & $(\mathrm{T}$ & $\mathrm{F} / \mathrm{V} / \mathrm{I}$ & $P$ & l) b \\
\hline OsSIP2;I ZmSIP2;I AtSIP2;I & $S$ & $\mathrm{H}$ & G & $\mathrm{A} / \mathrm{S}$ \\
\hline
\end{tabular}

a These selectivity filters are found only in Arabidopsis

bar/R selectivity filter reported by Wallace and Roberts [24]. 
Table 5: Ar/R signatures that are unique to rice and maize and are not found in Arabidopsis

\begin{tabular}{lcccc}
\hline MIP Members & H2 & H5 & LEI & LE2 \\
\hline TIP family & & & & \\
ZmTIP4;I, ZmTIP4;2 & H & S & A & R \\
OsTIP4;2, ZmTIP4;3 & Q & S/T & A & R \\
OsTIP5;I, ZmTIP5;I & Q & V & A & R \\
OsTIP4;। & T & T & A & R
\end{tabular}

\begin{tabular}{lllll}
\hline NIP family & & & & \\
OsNIP2;I, OsNIP2;2, ZmNIP2;I, ZmNIP2;2 & G & S & G & R \\
OsNIP3;2 & A & A & A & R \\
OsNIP4;I & C & G & G & R \\
OsNIP3;5 & A & A & P & R
\end{tabular}

\section{SIP family}

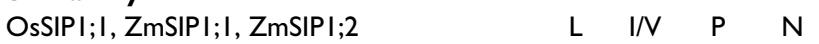

the plant MIPs, we first validated our approach by modeling the SoPIP2; 1 structure using the templates from bacteria and mammals and compared with the experimental structure determined at $2.1 \AA$ resolution (Figure 7; see Methods section). The $\alpha$-carbon backbone of the model generated using three templates shows an excellent agreement with the $\mathrm{x}$-ray structure in the TM region (six TM helices + loops B and E). The higher RMSD observed for the $\mathrm{C} \alpha$ atoms of the whole structure is due to the differences in the loops outside the TM region. Except SIPs, almost all the plant MIP sequences considered for this study have the characteristic sequence features typically found in aquaporin sequences (such as NPA motifs, conserved glutamates in TM1 and TM4 etc.).

Homology models of plant MIPs were characterized and structural subclasses were derived based on the residues forming the ar/R selectivity filter. The ar/R tetrad in PIP, NIP and TIP members from Arabidopsis obtained in this study are identical to that reported earlier by Wallace and Roberts [24](see above). They used the MOE homology
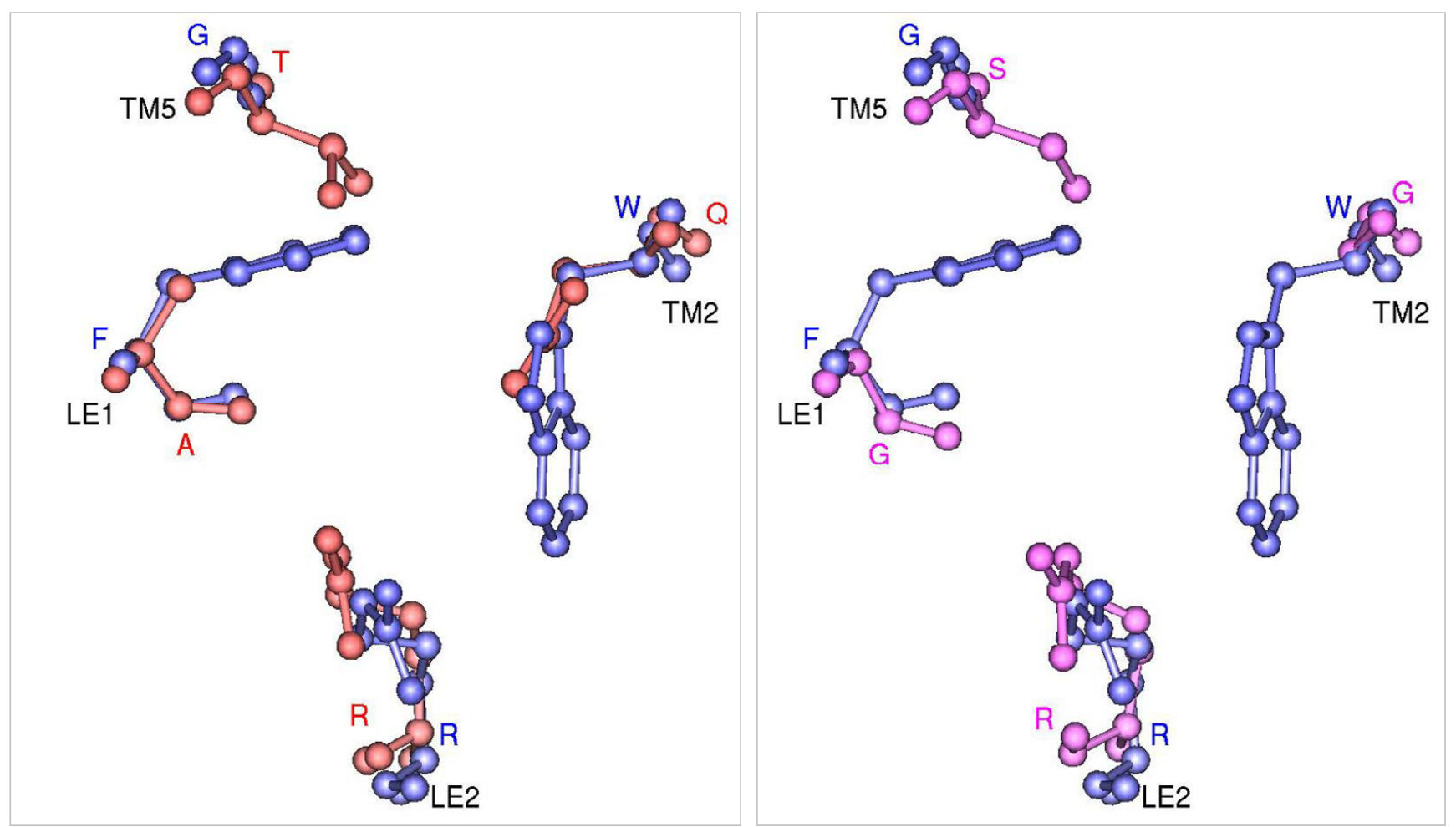

Figure 5

Ar/R selectivity filters of OsTIP4;2 and OsNIP2;I. Ar/R selectivity filter of water-specific OsTIP4;2 (red; left) and OsNIP2;I (pink; right) homology models. Transmembrane regions of both the models were first superposed individually on glycerol transporter GlpF (blue) and only the residues forming the ar/R tetrad from the superposed structures are shown in ball-and-stick model. Residue names in one letter code are given for OsTIP4;2 in red, for OsNIP2; in pink and for GlpF in blue. The transmembrane segments and the loop regions to which these residues belong are indicated. The projection shown for each filter is viewed perpendicular to the membrane plane from the extracellular side. 


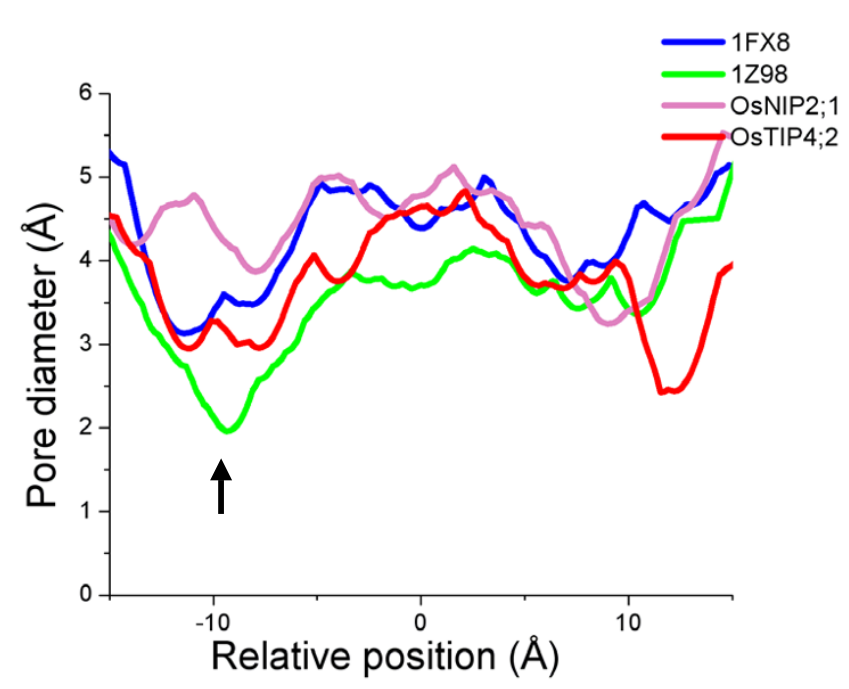

Figure 6

Comparison of pore radius profiles. Pore diameter profiles of water-specific SoPIP2; I (PDB ID: IZ98), glycerol-specific GlpF (PDB ID: IFX8), OsTIP4;2 and OsNIP2;I calculated using HOLE [63]. The black arrow indicates the approximate location of ar/R constriction region. The position $\mathrm{Z}=0 \AA$ corresponds to the location of NPA region.

program [53] based on a segment matching procedure [54]. In the present study, MODELLER [55] was used to build the homology models and SCWRL3 [56] was used to refine the side chains. Although MOE and MODELLER used different modeling strategies, the resultant models from two different approaches have clear agreement in the pore region.
The differences observed in the selectivity filters of one subgroup of SIP sequences in the two studies could be attributed to the fact that SIP sequences are the most diverse sequences. Many structurally important positions in GlpF and AQP1 have been shown to be different in SIPs [50]. For example, the conserved E17 (1J4N numbering) in TM1 is replaced by aspartate. Similarly, F24 in TM1 and Y99 in TM3 are replaced by Trp and Arg respectively. Our modeling approach indeed correctly aligned the residues E17, F24 and Y99 and the highly conserved Q103 in TM3 with the corresponding residues in SIP members (see Additional files 1, 2, 3, 4, 5, 6, 7, 8). However, Wallace and Roberts' method failed to align these positions correctly and several positions in the N-terminal half of SIP proteins are not correctly aligned (see supplementary data of [24]). Hence we believe that our alignment is more accurate and our model which resulted in a different selectivity filter for AtSIPs (Table 4) is more likely to be the correct one. Thus despite low sequence identities with the bacterial and mammalian template structures, the TM region of all the plant MIP homology models gives a reliable starting point to characterize the transmembrane helix packing and pore regions of four MIP subfamilies from three plant species.

\section{Small residues at helix-helix interfaces are strongly group- conserved in plant MIPs}

Analysis of high-resolution structures of 11 membrane and 23 soluble $\alpha$-helix bundle proteins revealed that high abundance of small residues (Gly, Ser, Thr and Ala) mediate helix-helix interactions in membrane proteins and result in closely packed helices [33]. The high propensity observed for small and weakly polar residues to occur in

Table 6: Percentage sequence identities between plant MIP subfamilies and the three template sequences

\begin{tabular}{|c|c|c|c|c|}
\hline Template $^{a}$ & PIPs & TIPs & NIPs & SIPs \\
\hline & \multicolumn{4}{|c|}{ Rice } \\
\hline AQPI & $38.2-44.8$ & $31.4-40.2$ & $25.9-34.7$ & $23.4-25.7$ \\
\hline GlpF & $31.0-35.4$ & $29.2-36.3$ & $25.5-38.5$ & $22.0-29.4$ \\
\hline \multirow[t]{2}{*}{ AqpZ } & $27.2-35.5$ & $30.1-38.3$ & $28.6-39.9$ & $26.7-29.1$ \\
\hline & \multicolumn{4}{|c|}{ Maize } \\
\hline AQPI & $40.1-46.0$ & $30.0-37.9$ & $29.0-32.2$ & $25.2-26.1$ \\
\hline GlpF & $31.6-34.5$ & $29.7-33.8$ & $32.3-35.5$ & $22.9-26.5$ \\
\hline \multirow[t]{2}{*}{ AqpZ } & $27.8-36.2$ & $30.8-38.1$ & $33.2-40.4$ & $24.3-26.0$ \\
\hline & \multicolumn{4}{|c|}{ Arabidopsis } \\
\hline AQPI & $40.9-45.3$ & $29.6-40.4$ & $26.6-33.2$ & $24.2-26.8$ \\
\hline GlpF & $29.2-35.3$ & $26.9-33.2$ & $32.3-39.3$ & $24.8-29.6$ \\
\hline$A q p Z$ & $29.4-34.4$ & $27.2-38.5$ & $32.6-39.2$ & $22.4-24.0$ \\
\hline
\end{tabular}

a Amino acid sequences corresponding to AQPI (bovine), GlpF (E. coli) and AqpZ (E. coli) were used for this analysis. The corresponding PDB codes of these proteins are IJ4N, IFX8 and IRC2 and they served as template structures for the plant MIP modeling. 


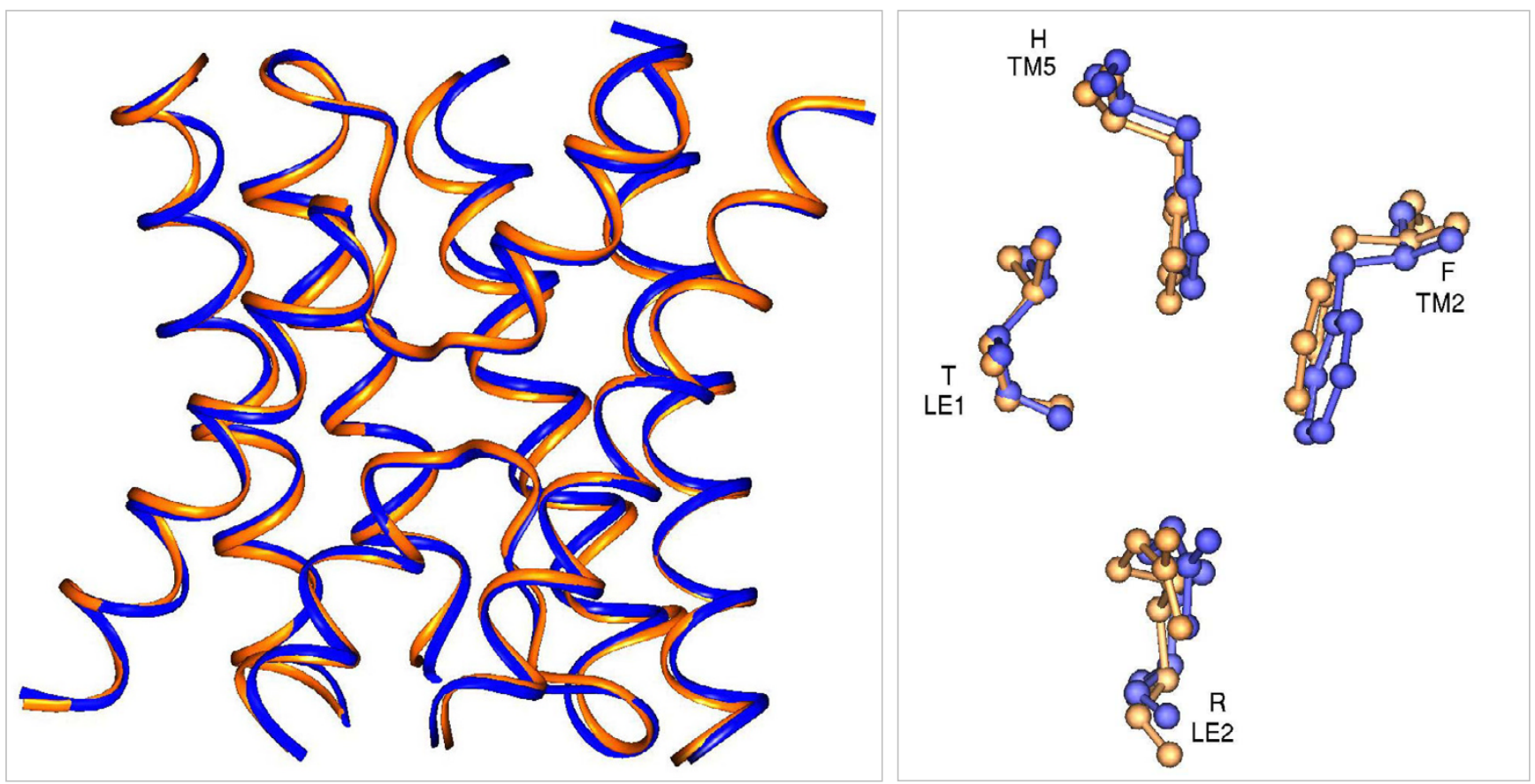

Figure 7

Comparison of X-ray and model structures of SoPIP2; I. Homology model generated for SoPIP2; I is superposed on the experimentally determined structure of SoPIP2;I. Left: Only the transmembrane helical regions and the loops B and E are shown. Right: Residues forming the Ar/R selectivity filters of modeled and the X-ray structures are shown after superposition in ball-and-stick representation. The transmembrane segments and the loop regions to which these residues belong are indicated. There is an excellent agreement between the modeled and the X-ray structures in the transmembrane region.

the closely packed interfaces give rise to motifs such as GxxxG that are known to drive transmembrane helix association [32,57]. Because of the lack of side chain, interfacial Gly residues can participate in weak $\mathrm{C} \alpha-\mathrm{H} . . . \mathrm{O}$ type of hydrogen bonds. The small size of the residues can also facilitate inter-helix interactions involving backbone $\mathrm{C}=$ $\mathrm{O}$ and N-H groups [32]. The functional groups of the Ser, Thr and Cys side-chains can also form inter-helical hydrogen bonds with the backbone $\mathrm{C}=\mathrm{O}$ and $\mathrm{N}-\mathrm{H}$ groups across the helix interface. It has been observed that the interfaces of transmembrane helix pairs in GlpF are lined by small and polar residues [33] and networks of $\mathrm{C} \alpha$ $\mathrm{H}$...O interactions were identified in the high-resolution crystal structure (PDB ID: 1FX8) of this protein [32]. Stroud et al. [18] have suggested that such weak hydrogen bonds could explain the stability of E. coli AqpZ in denaturing conditions. Close packing of helices due to the occurrences of small residues at the helix-helix interface has been shown to have functional significance. Recent experimental studies showed that anion permeability of mammalian AQP6 might be due to the substitution of interfacial glycine at TM2 by an Asn residue [58]. This Gly$>$ Asn substitution observed in AQP6 seems to give more flexibility to this protein compared to water-specific AQP1 and such flexibility is proposed to be one of the require- ments to convert a water-specific channel to anion permeable channel.

Structure-based sequence alignments have helped in identifying highly conserved active site residues [59,60]. In this study, analysis of sequence conservation in the structure-based sequence alignments of plant MIPs clearly showed that the small residues occurring in the transmembrane helix interfaces are very highly conserved as a group in plant MIPs. This conservation is seen for most of the small residues in the interface even in distantly related SIP families (see Additional files 1, 2, 3, 4, 5, 6, 7, 8). For any one amino acid type, the sequence identity may be 40\% (example, Gly 133 position in TM3; Table 3), but when the small and polar amino acids Gly, Ala, Ser, Thr and Cys are considered as a group, the conservation is close to $100 \%$. In the analysis of high-resolution crystal structures, Smith and his coworkers have shown that the molecular notches created by these small residues are a common element of the most closely packed helices in the core of membrane proteins $[33,49]$. In an analysis of 1047 class A GPCRs, it was revealed that small or polar residues are strongly group-conserved in helix-interface positions [49]. The same analysis was carried out in opsin, amine, olfactory and peptide GPCR subfamilies separately 
and it was shown that in some positions one or another amino acid of this group is strongly preferred in the subfamilies. In the present analysis of 105 plant MIP sequences, we have shown that the small or polar residues are strongly conserved in the helix-helix interfaces as a group. In the subfamily-specific analysis, we have also observed that in some positions, a specific amino acid of this group is preferred in the subfamilies. For example, position 256 is Ala in PIPs and TIPs and it is Gly in NIPs (Table 3 ). Similarly in position 55 , Thr is predominantly observed in PIPs while most of the other family members have Gly in this position. It should be noted this group conservation at the helix-helix interface would not have been recognized if we had compared just the sequences alone using the conventional multiple sequence alignment tools. In substitution matrices developed exclusively for transmembrane proteins [61,62], the substitution of Thr by Gly or vice versa is more unlikely. This clearly demonstrates the use of structure-based sequence alignment for diverse proteins belonging to the same family where the relationship is difficult to detect.

\section{Ar/R selectivity filters unique to rice and maize}

Nine rice and eight maize MIPs have ar/R signatures that seem to be distinct from any of the Arabidopsis MIPs (Table 5). Among the nine OsMIPs, eight have been shown to be expressed [43] and the maize MIPs are derived from the cDNA sequences [7]. The ar/ $\mathrm{R}$ tetrad of three rice and four maize TIP members (Table 5) is not found in Arabidopsis. The conserved feature of five of the members of this group (OsTIP4;1， OsTIP4;2, ZmTIP4;1，ZmTIP4;2 and ZmTIP4;3) is that the residue at $\mathrm{H} 2$ position is hydrophilic and the $\mathrm{H} 5$ residue is either serine or threonine. This gives rise to a wider hydrophilic constriction. Recent site-directed mutagenesis studies on rat AQP1 showed that replacement of Phe at $\mathrm{H} 2$ and His at $\mathrm{H} 5$ positions by Ala did not have any effect on water flux [23]. It was concluded that rat AQP1 water permeability is independent of the polarity at the ar/R constriction. However, the increase in constriction diameter did play a role in the mutant rat AQP1, facilitating the transport of bigger molecules like glycerol. Experimental studies have demonstrated that Arabidopsis TIP members have been shown to transport urea (AtTIP1;1, AtTIP1;2, AtTIP2;1 and AtTIP4;1) [8] and ammonia (AtTIP2;1 and AtTIP2;3) [10]. To our knowledge, no experimental data for the selectivity of the above rice and maize TIP members is presently available. The hydrophilic putative ar/R selectivity region for this group of TIP members (OsTIP4;1, OsTIP4;2, ZmTIP4;1, ZmTIP4;2 and ZmTIP4;3) with its wider constriction appears to be capable of transporting larger hydrophilic solutes similar to glycerol (Figure 5).

In the ar/R selectivity regions of five OsNIPs and two ZmNIPs (Table 5), all three positions (H2, H5 and LE1) are occupied by small residues, indicating that ar/R constriction of this group will be the largest among all the modeled plant MIPs. Our HOLE [63] analysis shows that the diameter at this constriction is more than $4.0 \AA$ that is $\sim 1.2 \AA$ larger than that of glycerol transporter, the largest known constriction among the experimentally determined structures. Experimental studies have shown that NIP members facilitate the transport of glycerol [12]. It has been speculated that plant aquaporins transport different solutes such as arsenite [64]. Rice and maize NIP members with small residues in three out of four positions of ar/R tetrad (Table 5) have the capability to conduct much larger solute molecules and thus will have distinct structural and functional features, representing a novel group of plant MIPs. This is supported by a recent study that identified a silicon transporter gene in rice [65]. This gene belongs to the aquaporin family and the protein amino acid sequence is identical to that of OsNIP2;1. The same study also suggested that other NIP members, ZmNIP2;1 and ZmNIP2;2, might also be involved in silicon uptake in maize.

A recent study on the two Arabidopsis NIP members demonstrated that the residue at $\mathrm{H} 2$ position is key in determining the selectivity of the channel [11]. Functional studies showed that AtNIP6;1 with Ala at $\mathrm{H} 2$ position exhibited extremely low water permeability but transported larger uncharged solutes like formamide, glycerol and urea. When Ala at $\mathrm{H} 2$ was substituted by Trp similar to the ar/R signature of soybean nodulin 26 , the archetype of the NIP subfamily, the mutant channel acquired the ability to facilitate water transport and prevented the transport of bulkier urea similar to the soybean nodulin 26 and other NIP members having this signature (Table 4 ). Due to the presence of a small residue at $\mathrm{H} 2$, the pore aperture at the ar/R region increased in NIP6; 1 . The larger diameter should have resulted in a pore with higher water permeability. Contrary to the expectations, this was not the case. A similar paradox was also observed by Stroud and his coworkers [22]. The ar/R regions in the crystal structures of AQP1, AqpM and GlpF constrict the diameter of the channels to $1.86 \AA, 2.54 \AA$ and $3.14 \AA$ respectively. Yet, the most efficient water channel is AQP1 and GlpF is a poor water-conducting glycerol channel. Conductance rate of water in AqpM is relatively low in comparison to AQP1. Thus the cross-sectional surface area of the $\mathrm{ar} / \mathrm{R}$ selectivity filter and the rate of conductance of water seem to be inversely correlated. A hypothesis based on thermodynamic considerations postulates that in pores with larger diameters, the channel may not be able to properly organize water at the ar/R region [11]. Computational studies have to be carried out to validate this hypothesis. With three of the four in the ar/R tetrad are small residues, in the NIP members like OsNIP2;1 identified in this study, the small residues essentially do not impose any 
constriction. We propose that these NIP members with diameter larger than that of GlpF in this region are likely to show the rate of water conductance that will be comparable to or even lower than that of GlpF (see above).

SIP subfamily is distantly related in sequence to the other MIP family members and many substitutions in the putative functional regions have been noticed in the analysis of SIP family of proteins [50]. Recent studies using membrane vesicles from yeast cells harbouring one of the Arabidopsis SIP members showed that both AtSIP1;1 and AtSIP1;2 have water channel activity [66]; AtSIP2;1 did not show any such activity although the protein was clearly present in the membrane vesicles. The biochemical characterization of the channel and the actual physiological functions remain to be determined for the SIPs. Homology modeling of SIP members shows that the ar/R filters have features that are very different from other MIP members. The conserved Arg at LE2 position is absent in all the SIPs. The challenges imposed due to the sequence divergence resulted in a slightly different model for AtSIP1;1 and AtSIP1;2 in our study (see above). While the model of Wallace and Roberts [24] contained a threonine at $\mathrm{H} 2$ position, our model has a hydrophobic residue at the same position. Sequence analysis and putative ar/R signatures suggest that SIPs are most likely to have substrate specificity very different from all known characterized MIPs.

\section{Conclusion}

We have structurally characterized rice aquaporins along with those from maize and Arabidopsis. Homology modeling studies were used to build structural models for 105 plant MIPs. Analysis of structure-based sequence alignment of plant MIPs showed that small and weakly polar residues have very high group conservation in the helixhelix interface. We propose that occurrence of small residue in the transmembrane helix interface enables close helix - helix interactions in the transmembrane region in MIP members. Homology models were used to identify the ar/R constriction in all three plant species. Structural characterization based on the ar/R signatures showed that TIP, NIP and SIP members from rice and maize have selectivity filters in the ar/R region that are not found in Arabidopsis. A subclass of NIP members has been found to have the constriction with the largest pore diameter since three of the four residues in the ar/ $\mathrm{R}$ tetrad are small. A recently discovered rice silicon transporter [65] belongs to this group. Members of this subgroup could thus represent a novel group of plant MIPs. SIP members with their unusual ar/R tetrad suggest that their substrate specificity could be very different from known characterized MIP genes. In summary, while the subfamilies diverge in the ar/ $\mathrm{R}$ signatures that can be directly related to the selectivity of the substrates, a strong conservation of small and polar residues at the helix-helix interfaces indicates that such group conservation is intended to keep the integrity of the "hour-glass" right-handed helical bundle structures in MIP family members. Now that the functional diversity of plant aquaporins has been recognized [67], characterization of novel plant MIPs, identification of new substrates that are transported by these proteins and mechanism of the transport will become the focus of future research that will eventually attempt to answer some of the important questions regarding the role of plant MIPs in root water uptake, reproduction or photosynthesis.

\section{Methods}

\section{Homology modeling of plant MIPs}

Modeling of rice, maize and Arabidopsis aquaporins was carried out in two stages. In the first stage, MODELLER $[55,68]$ software package (version $7 \mathrm{v} 7$ ) was used to construct homology models of all the three plant aquaporin proteins. MODELLER derives distance and dihedral angle restraints on the target sequence from its alignment with template 3-D structures and these relationships are expressed as conditional probability density functions. The spatial restraints thus derived and stereochemistry enforced by CHARMM22 force-field terms [69] are combined into an objective function and this function is minimized by an optimization procedure during model building. It has been shown that in MODELLER, using more than one template usually improves the quality of the model [70]. Hence for each of the plant MIP sequence, three high-resolution aquaporin structures [bovine AQP1 (PDB ID: $\underline{1 \mathrm{JN}}$; [16], E. coli GlpF (PDB ID: 1FX8; [17] and chain B of E. coli AQPZ (PDB ID: 1RC2; [18] were used as templates simultaneously in the comparative modeling procedure.

Pairwise sequence alignments between each plant MIP member and the three template sequences were carried out using the 'GAP' program available in the GCG package. Scoring matrix was BLOSUM62 and default values were used for all other parameters. The pairwise sequence identities between members of the MIP subfamilies and the three template sequences (AQP1, GlpF and AQPZ) range from 22.0 to $46 \%$ (Table 6 ). Among the subfamily members, the PIPs are the most closely related to the template sequences and the SIPs are the most distant family members. A multiple structural alignment based on iterative least-squares superposition technique was first carried out on the three template structures. Template sequences thus aligned based on the structural superposition were then used for aligning the target sequence. A dynamic programming method as implemented in MODELLER using "variable gap opening penalty" is used to align the target sequence with the template sequences. This gap penalty avoids placing gaps in secondary structural elements and 
favours gap in exposed regions and curved parts of the main-chain. Since sequence-structure alignment is a vital step in the model building process, we further checked the target-template alignment manually and gaps in the middle of the helices or in the conserved loops B or E were removed. The knowledge of strictly conserved residues in the transmembrane region reported in the aquaporin sequence analysis studies [71] has been used to further refine the target-template alignment. The residues E17 in TM1, Q103 in TM3, E144 in TM4 and P218 in TM6 (1J4N numbering) are highly conserved and hence alignments in these transmembrane segments have less ambiguity. In a few cases, the alignment was manually adjusted so that the conserved residues in the respective positions are brought under the same column. The default AS1 scoring matrix was used. The resultant alignment was given as input to MODELLER to build models with 'very fast' simulated annealing protocol. For each target sequence, 10 final models were created and the model with the lowest objective function value was selected. The loops of this model were further refined using MODELLER'S loop optimization procedure.

Prediction of side-chain conformation is an important component of the modeling procedure. In the second stage, we have used a side-chain prediction algorithm, SCWRL3 [56,72], on the MODELLER-generated structure. SCWRL3 uses graph theory to solve the combinatorial problem encountered in the side-chain refinement. This algorithm was used to build side-chains of the non-conserved residues on the backbone models generated by MODELLER. Finally, this model was subjected to 200 steps of steepest descent and 200 steps of conjugate gradient energy minimization methods using GROMACS $[73,74]$. The stereochemical quality of all the models was evaluated using PROCHECK $[75,76]$.

Analysis of the pore dimensions of MIP structural models has been carried out using the program HOLE $[63,77]$. In this algorithm, a Monte Carlo simulated annealing procedure is used that finds a best path for a sphere with a variable radius to squeeze through the channel. Initially, the model was superposed on the crystal structure of AQP1 (PDB ID: 1J4N) using the "Structure Alignment" option available in the "Homology" module of InsightII (Accelrys, San Diego, CA). This enables easier comparison of pore diameter profiles of different models and hence the coordinates of the superposed structure were used to analyze the pore dimensions. The initial point within the channel is taken as the average of $\mathrm{C} \alpha$ coordinates of the two conserved asparagines (Asn 78 and Asn 194 in 1 J4N numbering) and a third residue (Val 178 in $114 \mathrm{~N}$ or an equivalent residue in other models) from TM5 which is located on the channel wall opposite to the conserved asparagines. An initial vector of $\langle 0,0,-1\rangle$ was specified. AMBER [78]-based van der Waals radius file was used in the calculation of pore dimensions. Crystal structure studies [79] and molecular dynamics simulations [80] suggest that Arg side chain in LE2 position can exist in two distinct conformations, one that maintains a continuous single file of water molecules and the other that completely occludes the channel. It is suggested that alteration between the two conformations can regulate the open probability of the water pore and hence such a fluctuation is proposed to be a good candidate for a possible gating mechanism in aquaporins. In the HOLE calculations, our aim is to find the pore diameter in the open state of the channel. Hence in all the homology models of plant MIPs, the sidechain dihedral angles of Arg in LE2 position were constructed similar to that of water-specific AQP1 channel (PDB ID: 1J4N).

\section{Validity of the modeling protocol}

To test the validity of the approach, we constructed homology models of spinach plasma membrane aquaporin SoPIP2; 1 . The SoPIP2;1 sequence has 30-44\% identity with the three template sequences. A comparison of the experimentally determined structure showed that RMSD of C $\alpha$-trace is $4.16 \AA$ and if only the transmembrane helical regions are considered, it is $1.06 \AA$. In the generated SoPIP2;1 model, a slight improvement in the RMSD of transmembrane helices is seen after side chain refinement and minimization. The superposed experimental and model SoPIP2;1 structures are shown in Figure 7 . The helical backbone and the ar/R selectivity filter residues of the model structure are in excellent agreement with the X-ray structure. Small deviations are observed in the termini of transmembrane helices. It has been shown that use of more than one template structure generally increases the accuracy of the models [70]. Hence in the present study, three templates (PDB IDs: 1J4N, 1FX8 and $\underline{1 R C 2-B}$ chain) were chosen for modeling the plant MIPs.

\section{Authors' contributions}

$\mathrm{RS}$ conceived and designed the project. $\mathrm{AB}$ carried out the project. RS wrote the manuscript. Both the authors approved the final version of the manuscript.

\section{Additional material}

\section{Additional File 1}

Structure-based sequence alignment of plant MIPs in TM1 region. Structure-based sequence alignments are provided for all the 105 plant MIPs from the three plant species in the TM1 region. The first six sequences correspond to the experimentally determined aquaporin structures from different species. Their respective PDB IDs are shown in the first column. The beginning and end residue numbers are also given for each PDB structure. Small and weakly polar residues (Gly, Ala, Thr, Ser and Cys) occurring in the helix-helix interfaces are shaded in gray color. Click here for file

[http://www.biomedcentral.com/content/supplementary/14726807-7-27-S1.pdf] 


\section{Additional File 2}

Structure-based sequence alignment of plant MIPs in TM2 region. Structure-based sequence alignments are provided for all the 105 plant MIPs from the three plant species in the TM2 region. The first six sequences correspond to the experimentally determined aquaporin structures from different species. Their respective PDB IDs are shown in the first column. The beginning and end residue numbers are also given for each PDB structure. Small and weakly polar residues (Gly, Ala, Thr, Ser and Cys) occurring in the helix-helix interfaces are shaded in gray color. The residues forming arginine/aromatic selectivity filter are shown in bold.

Click here for file

[http://www.biomedcentral.com/content/supplementary/14726807-7-27-S2.pdf]

\section{Additional File 3}

Structure-based sequence alignment of plant MIPs in TM3 region. Structure-based sequence alignments are provided for all the 105 plant MIPs from the three plant species in the TM3 region. The first six sequences correspond to the experimentally determined aquaporin structures from different species. Their respective PDB IDs are shown in the first column. The beginning and end residue numbers are also given for each PDB structure. Small and weakly polar residues (Gly, Ala, Thr, Ser and Cys) occurring in the helix-helix interfaces are shaded in gray color Click here for file

[http://www.biomedcentral.com/content/supplementary/14726807-7-27-S3.pdf]

\section{Additional File 4}

Structure-based sequence alignment of plant MIPs in TM4 region. Structure-based sequence alignments are provided for all the 105 plant MIPs from the three plant species in the TM4 region. The first six sequences correspond to the experimentally determined aquaporin structures from different species. Their respective PDB IDs are shown in the first column. The beginning and end residue numbers are also given for each PDB structure. Small and weakly polar residues (Gly, Ala, Thr, Ser and Cys) occurring in the helix-helix interfaces are shaded in gray color. Click here for file

[http://www.biomedcentral.com/content/supplementary/14726807-7-27-S4.pdf]

\section{Additional File 5}

Structure-based sequence alignment of plant MIPs in TM5 region. Structure-based sequence alignments are provided for all the 105 plant MIPs from the three plant species in the TM5 region. The first six sequences correspond to the experimentally determined aquaporin structures from different species. Their respective PDB IDs are shown in the first column. The beginning and end residue numbers are also given for each PDB structure. Small and weakly polar residues (Gly, Ala, Thr, Ser and Cys) occurring in the helix-helix interfaces are shaded in gray color The residues forming arginine/aromatic selectivity filter are shown in bold.

Click here for file

[http://www.biomedcentral.com/content/supplementary/14726807-7-27-S5.pdf]

\section{Additional File 6}

Structure-based sequence alignment of plant MIPs in TM6 region. Structure-based sequence alignments are provided for all the 105 plant MIPs from the three plant species in the TMG region. The first six sequences correspond to the experimentally determined aquaporin structures from different species. Their respective PDB IDs are shown in the first column. The beginning and end residue numbers are also given for each PDB structure. Small and weakly polar residues (Gly, Ala, Thr, Ser and Cys) occurring in the helix-helix interfaces are shaded in gray color. Click here for file

[http://www.biomedcentral.com/content/supplementary/14726807-7-27-S6.pdf]

\section{Additional File 7}

Structure-based sequence alignment of plant MIPs in the loop B region. Structure-based sequence alignments are provided for all the 105 plant MIPs from the three plant species in the loop B region. The first six sequences correspond to the experimentally determined aquaporin structures from different species. Their respective PDB IDs are shown in the first column. The beginning and end residue numbers are also given for each PDB structure. Small and weakly polar residues (Gly, Ala, Thr, Ser and Cys) occurring in the helix-helix interfaces are shaded in gray color. Click here for file

[http://www.biomedcentral.com/content/supplementary/14726807-7-27-S7.pdf]

\section{Additional File 8}

Structure-based sequence alignment of plant MIPs in the loop E region. Structure-based sequence alignments are provided for all the 105 plant MIPs from the three plant species in the loop E region. The first six sequences correspond to the experimentally determined aquaporin structures from different species. Their respective PDB IDs are shown in the first column. The beginning and end residue numbers are also given for each PDB structure. Small and weakly polar residues (Gly, Ala, Thr, Ser and Cys) occurring in the helix-helix interfaces are shaded in gray color. The residues forming arginine/aromatic selectivity filter are shown in bold.

Click here for file

[http://www.biomedcentral.com/content/supplementary/14726807-7-27-S8.pdf]

\section{Acknowledgements}

We thank Prof. Andrej Sali for providing the MODELLER package and Prof. Ronald Dunbrack Jr. for making the program SCWRL3 available. Dr. Oliver Smart is acknowledged for his HOLE program. We thank all the three anonymous reviewers for their valuable comments and suggestions. This project is funded by a grant from the Ministry of Human Resources Development (MHRD), Government of India. Anjali Bansal acknowledges Council of Scientific and Industrial Research (CSIR), India for a senior research fellowship. We thank members of our laboratory for discussions.

\section{References}

I. Agre P, Kozono D: Aquaporin water channels: molecular mechanisms for human diseases. FEBS Lett 2003, 555:72-78.

2. Heymann JB, Engel A: Aquaporins: phylogeny, structure, and physiology of water channels. News Physiol Sci I999, I 4:187-193.

3. Maurel C: Aquaporins and water permeability of plant membranes. Ann Rev Plant Physiol Plant Mol Biol 1997, 48:399-429.

4. Tyerman SD, Niemietz CM, Bramley H: Plant aquaporins: multifunctional water and solute channels with expanding roles. Plant Cell and Environ 2002, 25:173-194. 
5. Johansson I, Karlsson M, Johanson U, Larsson C, Kjellbom P: The role of aquaporins in cellular and whole plant water balance. Biochim Biophys Acta 2000, I 465:324-342.

6. Johanson U, Karlsson M, Johansson I, Gustavsson S, Sjovall S, Fraysse L, Weig AR, Kjellbom P: The complete set of genes encoding major intrinsic proteins in Arabidopsis provides a framework for a new nomenclature for major intrinsic proteins in plants. Plant Physiol 200 I, I 26: I358-1369.

7. Chaumont F, Barrieu F, Wojcik E, Chrispeels MJ, Jung R: Aquaporins constitute a large and highly divergent protein family in maize. Plant Physiol 200I, I 25: I206-1215.

8. Liu LH, Ludewig U, Gassert B, Frommer WB, von Wiren N: Urea transport by nirogen-regulated tonoplast intrinsic proteins in Arabidopsis. Plant Physiol 2003, 133:1 220-1228.

9. Jahn TP, Moller ALB, Zeuthen T, Holm LM, Klaerke DA, Mohsin B, Kuhlbrandt W, Schjoerring JK: Aquaporin homologues in plants and mammals transport ammonia. FEBS Lett 2004, 574:3I-36.

10. Loque D, Ludewig U, Yuan L, von Wiren N: Tonoplast intrinsic proteins AtTIP2; I and AtTIP2;3 faciliate NH3 transport into the vacuole. Plant Physiol 2005, 137:67|-680.

II. Wallace IS, Roberts DM: Distinct transport selectivity of two structural subclasses of the nodulin-like intrinsic protein family of plant aquaglyceroporin channels. Biochemistry 2005 , 44:16826-16834

12. Wallace IS, Wills DM, Guenther JF, Roberts DM: Functional selectivity for glycerol of the nodulin $\mathbf{2 6}$ subfamily of plant membrane intrinsic proteins. FEBS Lett 2002, 523:109-I I2.

13. Uehlein N, Lovisolo C, Siefritz F, Kaldenhoff R: The tobacco aquaporin NtAQPI is a membrane $\mathrm{CO} 2$ pore with physiological functions. Nature 2003, 425:734-737.

14. Stroud RM, Savage D, Miercke LJW, Lee JK, Khademi S, Harries W: Selectivity and conductance among the glycerol and water conducting aquaporin family of channels. FEBS Lett 2003 , 555:79-84

15. Protein Data Bank [http://www.pdb.org/].

16. Sui H, Han BG, Lee JK, Walian P, Jap BK: Structural basis of waterspecific transport through the AQPI water channel. Nature 200I, 4 I 4:872-878.

17. Fu D, Libson A, Miercke LJW, Weitzman C, Nollert P, Krucinski J, Stroud RM: Structure of a glycerol-conducting channel and the basis for its selectivity. Science 2000, 290:48I-486

18. Savage DF, Egea PF, Robles-Colmenares Y, O'Connell III JD, Stroud RM: Architecture and selectivity in aquaporins: 2.5 A structure of aquaporin Z. PLoS Biol 2003, I:334-340.

19. Gonen T, Sliz P, Kistler J, Cheng Y, Walz T: Aquaporin-0 membrane junctions reveal the structure of a closed water pore. Nature 2004, 429: 193-197.

20. Harries WEC, Akhavan D, Miercke LJW, Khademi S, Stroud RM: The channel architecture of aquaporin 0 at a 2.2-A resolution. Proc Natl Acad Sci U S A 2004, I O I: | 4045-I 4050.

2I. Tornroth-Horsefield S, Wang Y, Hedfalk K, Johanson U, Karlsson M, Tajkhorshid E, Neutze R, Kjellbom P: Structural mechanism of plant aquaporin gating. Nature 2006, 439:688-694.

22. Lee JK, Kozono D, Remis J, Kitagawa Y, Agre P, Stroud RM: Structural basis for conductance by the archaeal aquaporin AqpM at I.68 A. Proc Natl Acad Sci U S A 2005, 102:18932-18937.

23. Beitz E, Wu B, Holm LM, Schultz JE, Zeuthen T: Point mutations in the aromatic/arginine region in aquaporin I allow passage of urea, glycerol, ammonia and protons. Proc Natl Acad Sci U S A 2006, 103:269-274.

24. Wallace IS, Roberts DM: Homology modeling of representative subfamilies of Arabidopsis major intrinsic proteins: Classification based on the Aromatic/Arginine selectivity filter. Plant Physiol 2004, 135: 1059-1068.

25. de Groot BL, Grubmuller H: The dynamics and energetics of water permeation and proton exclusion in aquaporins. Curr Opin Struct Biol 2005, 15:176-183.

26. de Groot BL, Grubmuller H: Water permeation across biological membranes: Mechanism and dynamics of aquaporin-I and GlpF. Science 200I, 294:2353-2357.

27. Tajkhorshid E, Nollert P, Jensen MO, Miercke LJW, O'Connell III JD, Stroud RM, Schulten K: Control of the selectivity of the aquaporin water channel family by global orientational tuning. Science 2002, 296:525-530.

28. Chakrabarti N, Roux B, Pomes R: Structural determinants of proton blockage in aquaporins. J Mol Biol 2004, 343:493-5I0.
29. International Rice Genome Sequencing Project. The mapbased sequence of the rice genome. Nature 2005, 436:793-800.

30. Yu J, Hu S, Wang J, Wong GK, Li S, Liu B, Deng Y, Dai L, Zhou Y, Zhang $X$ : A draft sequence of the rice genome (Oryza sativa L ssp. indica). Science 2002, 296:79-92.

31. Goff SA, Ricke D, Lan TH, Presting G, Wang R, Dunn M, Glazebrook J, Sessions A, Oeller $\mathrm{P}$, Varma $\mathrm{H}$ : A draft sequence of the rice genome (Oryza sativa L.ssp japonica). Science 2002, 296:92-100

32. Senes A, Ubarretxena-Belandia I, Engelman DM: The C $\alpha-\mathbf{H}$...O hydrogen bond: A determinant of stability and specificity in transmembrane helix interactions. Proc Natl Acad Sci U S A 200 I, 98:9056-906I.

33. Eilers $M$, Patel $A B$, Liu W, Smith SO: Comparison of helix interactions in membrane and soluble $\alpha$-bundle proteins. Biophys J 2002, 82:2720-2736

34. Altschul SF, Madden TL, Schaffer AA, Zhang J, Zhang Z, Miller W, Lipman D]: Gapped BLAST and PSI-BLAST: a new generation of protein database search programs. Nucleic Acids Res 1997, 25:3389-3402.

35. TBLASTN at NCBI [http://www.ncbi.nlm.nih.gov:80/blast/ blast.cgi].

36. TBLASTN at RGP [http://riceblast.dna.affrc.go.jp/]

37. TBLASTN at TIGR [http://tigrblast.tigr.org/euk-blast/ index.cgi?project=osa I]

38. Benson DA, Karsch-Mizrachi I, Lipman DJ, Ostell J, Wheeler DL: GenBank. Nucleic Acids Res 2006, 34:D 16-D20.

39. GenBank [http://www.ncbi.nlm.nih.gov/Genbank/]

40. Rice Genome Project (RGP) [http://rgp.dna.affrc.go.jp/].

4I. Yuan Q, Ouyang S, Liu J, Suh B, Cheung F, Sultana R, Lee D, Quackenbush J, Buell RC: The TIGR rice genome annotation resource: annotating the rice genome and creating resources for plant biologists. Nucleic Acids Res 2003, 3I:229-233.

42. The Institute of Genomic Research (TIGR) [http:// www.tigr.org/tdb/e2kl/osal/].

43. Sakurai J, Ishikawa F, Yamaguchi T, Uemura M, Maeshima M: Identification of 33 rice aquaporin genes and analysis of their expression and function. Plant Cell Physiol 2005, 46: I568-I577.

44. Borodovsky M, Mclninch J: GenMark - Parallel gene recognition algorithm for both DNA strands. Comput \& Chem 1993, I7:123-133.

45. Mulder NJ, Apweiler R, Attwood TK, Bairoch A, Bateman A, Binns D, Bork P, Buillard V, Cerutti L, Copley R, Courcelle E, Das U, Daugherty L, Dibley M, Finn R, Fleischmann W, Gough J, Haft D, Hulo N, Hunter S, Kahn D, Kanapin A, Kejariwal A, Labarga A, LangendijkGenevaux PS, Lonsdale D, Lopez R, Letunic I, Madera M, Maslen J, McAnulla C, McDowall J, Mistry J, Mitchell A, Nikolskaya AN, Orchard S, Orengo C, Petryszak R, Selengut JD, Sigrist CJA, Thomas $\mathrm{PD}$, Valentin $\mathrm{F}$, Wilson $\mathrm{D}$, Hu CH, Yeats C: InterPro, progress and status in 2005. Nucleic Acids Res 2005, 33:D20I-D205.

46. InterPro [http://www.ebi.ac.uk/interpro/]

47. Karkouri KE, Gueune H, Delamarche C: MIPDB: a relational database dedicated to MIP family proteins. Biol Cell 2005, 97:535-543

48. MIPDB [http://genoweb.univ-rennes I.fr/Prot/index.html]

49. Liu $W$, Eilers $M$, Patel $A B$, Smith SO: Helix packing moments reveal diversity and conservation in membrane protein structure. J Mol Biol 2004, 337:713-729.

50. Johanson U, Gustavsson S: A new subfamily of major intrinsic proteins in plants. Mol Biol Evol 2002, 19:456-46।.

51. Madhusudhan MS, Marti-Renom MA, Eswar N, John B, Karchin R, Shen MY, Sali A: Comparative protein structure modeling. The Proteomics Protocols Handbook 2005:83 I-860.

52. Law RJ, Sansom MSP: Homology modelling and molecular dynamics simulations: comparative studies of human aquaporin-I. Eur Biophys J 2004, 33:477-489.

53. Kelly K: Multiple sequence and structural alignment in MOE. Chemical Computing Group. [http://www.chemcomp.com/ journal/align.htm]. 1996

54. Levitt M: Accurate modeling of protein conformation by automatic segment matching. J Mol Biol 1992, 226:507-533.

55. Sali A, Blundell TL: Comparative protein modeling by satisfaction of spatial restraints. J Mol Biol 1993, 234:779-8I5. 
56. Canutescu AA, Shelenkov AA, Dunbrack Jr. RL: A graph-theory algorithm for rapid protein side-chain prediction. Protein Sci 2003, I 2:200I-20I4.

57. Senes A, Gerstein M, Engelman DM: Statistical analysis of amino acid patterns in transmembrane helices: The GxxxG motif occurs frequently and in association with $\beta$-branched residues at neighboring positions. J Mol Biol 2000, 296:92I-936.

58. Liu K, Kozono D, Kato Y, Agre P, Hazama A, Yasui M: Conversion of aquaporin 6 from an anion channel to a water-selective channel by a single amino acid substitution. Proc Natl Acad Sci U S A 2005, 102:2192-2197.

59. Chen LQ, DiGiammarino E, Zhou XYE, Wang YJ, Toh D, Hodge TW Meehan EJ: High resolution crystal structure of human Rab9 GTPase - A novel antiviral drug target. J Biol Chem 2004 279:40204-40208.

60. Benach J, Atrian S, Gonzalez-Duarte R, Ladenstein R: The refined crystal structure of Drosophila lebanonensis alcohol dehy drogenase at 1.9 angstrom resolution. J Mol Biol 1998 282:383-399.

61. Ng PC, Henikoff JG, Henikoff S: PHAT: a transmembrane-specific substitution matrix. Bioinformatics 2000, I6:760-766.

62. Jones DT, Taylor WR, Thornton JM: A mutation data matrix for transmembrane proteins. FEBS Lett 1994, 339:269-275.

63. Smart OS, Neduvelil JG, Wang X, Wallace BA, Sansom MSP: HOLE: A program for the analysis of the pore dimensions of ion channel structural models. I Mol Graphics 1996, I 4:354-360.

64. Meharg AA, Jardine L: Arsenite transport into paddy rice (Oryza sativa) roots. New Phytologist 2003, I 57:39-44.

65. Ma JF, Tamai K, Yamaji N, Mitani N, Konishi S, Katsuhara M, Ishiguro M, Murata $Y$, Yano M: A silicon transporter in rice. Nature 2006 440:688-69l.

66. Ishikawa F, Suga S, Uemura T, Sato MH, Maeshima M: Novel type aquaporin SIPs are mainly localized to the ER membrane and show cell-specific expression in Arabidopsis thaliana. FEBS Lett 2005, 579:58|4-5820.

67. Kaldenhoff $\mathrm{R}$, Flscher $\mathrm{M}$ : Functional aquaporin diversity in plants. Biochim Biophys Acta 2006, I 758: | | 34- | | 4 I.

68. MODELLER [http://salilab.org/modeller].

69. Brooks BR, Bruccoleri RE, Olafson BD, States DJ, Swaminathan S, Karplus M: CHARMM: A program for macromolecular energy minimization and dynamics calculations. J Comp Chem 1983, 4:|87-2|17.

70. Sanchez R, Sali A: Evaluation of comparative protein structure modeling by MODELLER-3. Proteins: Struct Func Genetics 1997 Suppl. I:50-58.

7I. Heymann JB, Engel A: Structural clues in the sequences of the aquaporins. J Mol Biol 2000, 295: 1039-1053.

72. SCWRL3 [http://dunbrack.fccc.edu/SCWRL3.php]

73. Lindahl E, Hess B, van der Spoel D: GROMACS 3.0: a package for molecular simulation and trajectory analysis. I Mol Modeling 2001, 7:306-317.

74. GROMACS [http://www.gromacs.org/].

75. Laskowski RA, MacArthur MW, Moss DS, Thornton JM: PROCHECK-A program to check the stereochemical quality of protein structures. J Appl Cryst 1993, 26, Part 2:283-29I.

76. PROCHECK [http://www.biochem.ucl.ac.uk/ roman/procheck/procheck.html].

77. HOLE [http://hole.biop.ox.ac.uk/hole].

78. Weiner S], Kollman PA, Case DA, Singh UC, Ghio C, Alagona G, Profeta S, Weiner P: A new force-field for molecular mechanical simulation of nucleic acids and proteins. J Am Chem Soc 1984, 1 06:765-784.

79. Jiang J, Daniels BV, Fu D: Crystal structure of AqpZ tetramer reveals two distinct Arg- 189 conformations associated with water permeation through the narrowest constriction of the water-conducting channel. J Biol Chem 2006, 28 I :454-460.

80. Wang Y, Schulten K, Tajkhorshid E: What makes an aquaporin channel a glycerol channel? A comparative study of AqpZ and GIpF. Structure 2005, I3: | | 07- I | | 8 .

81. Notredame C, Higgins DG, Heringa J: T-Coffee: A novel method for fast and accurate multiple sequence alignment. J Mol Biol 2000, 302:205-217.
Publish with Biomed Central and every scientist can read your work free of charge

"BioMed Central will be the most significant development for disseminating the results of biomedical research in our lifetime. "

Sir Paul Nurse, Cancer Research UK

Your research papers will be:

- available free of charge to the entire biomedical community

- peer reviewed and published immediately upon acceptance

- cited in PubMed and archived on PubMed Central

- yours - you keep the copyright

Submit your manuscript here:

http://www.biomedcentral.com/info/publishing_adv.asp
BioMedcentral 